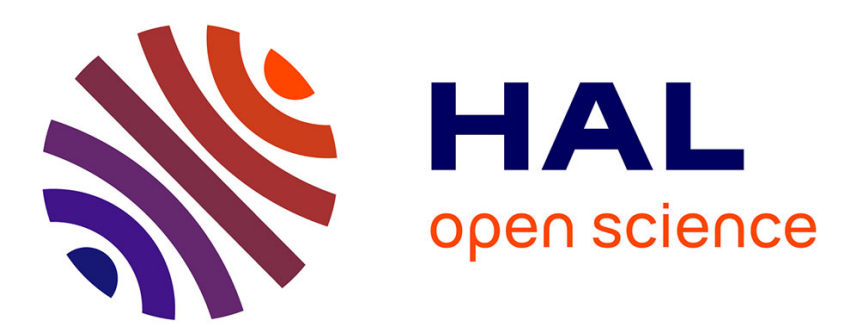

\title{
A posteriori error estimators for the fully discrete time dependent Stokes problem with some different boundary conditions
}

Fida El Chami, Toni Sayah

\section{- To cite this version:}

Fida El Chami, Toni Sayah. A posteriori error estimators for the fully discrete time dependent Stokes problem with some different boundary conditions. 2009. hal-00440440v1

\section{HAL Id: hal-00440440 \\ https://hal.science/hal-00440440v1}

Preprint submitted on 11 Dec 2009 (v1), last revised 15 Sep 2010 (v2)

HAL is a multi-disciplinary open access archive for the deposit and dissemination of scientific research documents, whether they are published or not. The documents may come from teaching and research institutions in France or abroad, or from public or private research centers.
L'archive ouverte pluridisciplinaire HAL, est destinée au dépôt et à la diffusion de documents scientifiques de niveau recherche, publiés ou non, émanant des établissements d'enseignement et de recherche français ou étrangers, des laboratoires publics ou privés. 


\title{
A POSTERIORI ERROR ESTIMATORS FOR THE FULLY DISCRETE TIME DEPENDENT STOKES PROBLEM WITH SOME DIFFERENT BOUNDARY CONDITIONS
}

\author{
FIDA EL CHAMI ${ }^{\dagger}$ AND TONI SAYAH SH $^{\ddagger}$
}

\begin{abstract}
In this paper we study the time dependent Stokes problem with some different boundary conditions. We establish a decoupled variational formulation into a system of velocity and a Poisson equation for the pressure. Hence, the velocity is approximated with curl conforming finite elements in space and Euler scheme in time and the pressure with standard continuous elements in space and Euler scheme in time. Finally, we establish optimal a priori and a posteriori estimates
\end{abstract}

Keywords Partial differential equations, a piori and a posteriori errors, time dependent Stokes equations.

\section{INTRODUCTION.}

This paper is devoted to the numerical solution of the time dependent Stokes equations for an incompressible fluid

with the incompressibility condition

$$
\left.\frac{\partial \mathbf{u}}{\partial t}-\nu \Delta \mathbf{u}+\nabla p=\mathbf{f} \quad \text { in } \Omega \times\right] 0, T[
$$

$$
\operatorname{div} \mathbf{u}=0 \quad \text { in } \Omega \times] 0, T[,
$$

the initial condition

the boundary conditions

$$
\mathbf{u}(0)=\mathbf{0} \quad \text { in } \Omega,
$$

$$
\mathbf{u} \times \mathbf{n}=0, \quad p=0 \quad \text { on } \partial \Omega \times] 0, T[,
$$

or

$$
\mathbf{u . n}=0, \quad \operatorname{curl} \mathbf{u} \times \mathbf{n}=0 \quad \text { on } \partial \Omega \times] 0, T[
$$

where $] 0, T\left[\right.$ is a given time-interval, $\Omega$ is a convex bounded simply connected domain of $\mathbb{R}^{3}$ with a polyhedral connected boundary $\Gamma=\partial \Omega$ and $\mathbf{n}$ the exterior unit normal to $\Gamma, \mathbf{u}$ the velocity and $p$ the pressure. All the quantities are taken at the point $(x, t)$ where $x=\left(x_{i}\right)_{1 \leq i \leq 3} \in \mathbb{R}^{3}$ denotes the position and $t \in[0, T]$ the time. $\mathbf{f}$ denotes the external forces applied to the fluid and $\nu>0$ is the viscosity.

These sets of boundary conditions lend themselves readily to a variational formulation where the Laplacian operator is expressed by a (curl, curl) term and the incompressibility condition by an equation of the form $(\nabla q, \mathbf{v})$. This suggests to use a partially non-confirming finite element method, where just the curl of the velocity is continuous at interface boundaries whereas the pressure is globally continuous.

We refer also to Girault's work [17] for a vector potential-vorticity approximation of similar Navier-Stokes type problems and to [16] for the steady-state incompressible Navier-Stokes equations with non standard boundary conditions. We also refer to [25] where Repin establishes a posteriori estimates for the velocity, stress and pressure fields for the stationary Stokes problem and where his approach is based on duality

\footnotetext{
December 10, 2009

† Faculté des Sciences II, Université Libanaise, Département de mathématiques, B.P. 90656, Fanar-Maten, Liban.

$\ddagger$ (email : tsayah@fs.usj.edu.lb) Faculté des Sciences, Université Saint-Joseph, B.P 11-514 Riad El Solh, Beyrouth 1107 2050, Liban.
} 
theory of the calculus of variations. A posteriori estimates for the Stokes problem and for some viscous flow problems were studied by a number of authors, [4], [30], [31], [9], [22] and [23]. Typically, they have been obtained in the frame of the so-called "residual method" originally proposed in [2] and [3] for the finite element approximations. For the a piori estimations of the Stokes problem, we can also cite the works of S. Repin [26], [27],[28]. Finally, in [1], we treat the same problem in stationary case.

In this work, we treat the time dependent stokes equations with some different boundary conditions. We establish a decoupled variational formulation into a system of velocity and a Poisson equation for the pressure. We introduce a fully discrete system using the curl conforming finite elements for the velocity and the standard continuous elements for the pressure in space, and the Euler scheme in time. Finally, we establish an optimal a priori and a posteriori error estimates.

\section{Description AND ANAlysis of the MODEL}

We denote by (Problem 1$)$ the system of equations (1.1), (1.2), (1.3) and (1.4), and by (Problem2) the system of equations (1.1), (1.2) (1.3) and (1.5). In all the paper, we denote by $C$ and $c$ generic positive constants.

In order to write the variational formulation of the previous problems, we introduce some spaces:

$$
\begin{aligned}
& W^{m, p}(\Omega)=\left\{v \in L^{p}(\Omega), \partial^{\alpha} v \in L^{p}(\Omega), \quad \forall|\alpha| \leq m\right\}, \\
& H^{m}(\Omega)=W^{m, 2}(\Omega),
\end{aligned}
$$

equipped with the following semi-norm and norm :

$$
|v|_{m, p, \Omega}=\left\{\sum_{|\alpha|=m} \int_{\Omega}\left|\partial^{\alpha} v(x)\right|^{p} d x\right\}^{1 / p} \quad \text { and } \quad\|v\|_{m, p, \Omega}=\left\{\sum_{k \leq m}|v|_{k, p, \Omega}^{p}\right\}^{1 / p}
$$

As usual, we shall omit $p$ when $p=2$ and denote by $(\cdot, \cdot)$ the scalar product of $L^{2}(\Omega)$. Also, recall the familiar notation :

with the Poincaré inequality

$$
H_{0}^{1}(\Omega)=\left\{v \in H^{1}(\Omega) ; v=0 \text { on } \Gamma\right\}
$$

$$
\forall v \in H_{0}^{1}(\Omega) ; \quad\|v\|_{0, \Omega} \leq C|v|_{1, \Omega} .
$$

Finally, we introduce the spaces :

$$
\begin{aligned}
& H(\operatorname{div}, \Omega)=\left\{\mathbf{v} \in L^{2}(\Omega)^{3}, \operatorname{div} \mathbf{v} \in L^{2}(\Omega)\right\} ; \quad H_{0}(\operatorname{div}, \Omega)=\{\mathbf{v} \in H(\operatorname{div}, \Omega), \mathbf{v} \cdot \mathbf{n}=0 \text { on } \Gamma\} \\
& H(\mathbf{c u r l}, \Omega)=\left\{\mathbf{v} \in L^{2}(\Omega)^{3}, \mathbf{c u r l} \mathbf{v} \in L^{2}(\Omega)^{3}\right\} ; \quad H_{0}(\mathbf{c u r l}, \Omega)=\{\mathbf{v} \in H(\mathbf{c u r l}, \Omega), \mathbf{v} \times \mathbf{n}=0 \text { on } \Gamma\}
\end{aligned}
$$

normed respectively by :

$$
\begin{aligned}
& \|\mathbf{v}\|_{H(\operatorname{div}, \Omega)}=\left\{\|\mathbf{v}\|_{0, \Omega}^{2}+\|\operatorname{div} \mathbf{v}\|_{0, \Omega}^{2}\right\}^{1 / 2}, \\
& \text { and } \\
& \|\mathbf{v}\|_{H(\operatorname{curl}, \Omega)}=\left\{\|\mathbf{v}\|_{0, \Omega}^{2}+\|\operatorname{curl} \mathbf{v}\|_{0, \Omega}^{2}\right\}^{1 / 2} .
\end{aligned}
$$

For the theoretical fondations of this spaces, we can refer to Duvaut \& Lions [13] and for the following regularity theorems, we refer to Bernardi [8], Dauge [11], Girault \& Raviart [15], Grisvard [18] and Nedelec [21].

Lemma 2.1. For each $\mathbf{g} \in L^{2}(\Omega)$, there exists a unique solution $\mathbf{w}$ in $H^{1}(\Omega) / \mathbb{R}\left(\right.$ resp. $\left.H_{0}^{1}(\Omega)\right)$ such that :

$$
(\nabla \mathbf{w}, \nabla \mathbf{v})=(\mathbf{g}, \nabla \mathbf{v}) \quad \forall \mathbf{v} \in H^{1}(\Omega) / \mathbb{R} \quad\left(\operatorname{resp} H_{0}^{1}(\Omega)\right)
$$


and there exists a positive constant $C$ such that:

$$
\|\mathbf{w}\|_{1, \Omega} \leq C\|\mathbf{g}\|_{0, \Omega} .
$$

Theorem 2.2. All functions $\mathbf{v} \in L^{2}(\Omega)^{3}$ satisfying :

$$
\operatorname{div} \mathbf{v}=0, \quad \operatorname{curl} \mathbf{v} \in L^{2}(\Omega)^{3}, \quad \mathbf{v} \cdot \mathbf{n}=0 \text { or } \mathbf{v} \times \mathbf{n}=0 \text { on } \Gamma
$$

belong to $H^{1}(\Omega)$ and we have

$$
\|\mathbf{v}\|_{1, \Omega} \leq C\|\mathbf{c u r l} \mathbf{v}\|_{0, \Omega}
$$

As usual, for handling time-dependent problems, it is convenient to consider functions defined on a time interval $] a, b\left[\right.$ with values in a functional space, say $X$. More precisely, let $\|\cdot\|_{X}$ denote the norm of $X$; then for any $r, 1 \leq r \leq \infty$, we define

$$
L^{r}(a, b ; X)=\{f \text { mesurable in }] a, b\left[; \int_{a}^{b}\|f(t)\|_{X}^{r} d t<\infty\right\}
$$

equipped with the norm

$$
\|f\|_{L^{r}(a, b ; X)}=\left(\int_{a}^{b}\|f(t)\|_{X}^{r} d t\right)^{1 / r},
$$

with the usual modifications if $r=\infty$. It is a Banach space if $X$ is a Banach space.

In view of the relation :

$$
-\Delta \mathbf{u}=\operatorname{curl} \text { curl } \mathbf{u} \quad(\text { as we have } \operatorname{div} \mathbf{u}=0)
$$

we can easily prove the following theorems.

Theorem 2.3. Let $\mathbf{f} \in L^{\infty}\left(0, T ; L^{2}(\Omega)^{3}\right)$.

(Problem1) has the following week variational formulation :

$$
\begin{aligned}
& \text { Find } \mathbf{u}(t) \in H_{0}(\mathbf{c u r l}, \Omega) \text { and } p(t) \in H_{0}^{1}(\Omega) \text { such that } \\
& \left(\frac{d}{d t} \mathbf{u}(t), \mathbf{v}\right)+\nu(\operatorname{curl} \mathbf{u}(t), \operatorname{curl} \mathbf{v})+(\nabla p(t), \mathbf{v})=(\mathbf{f}(t), \mathbf{v}) \quad \forall \mathbf{v} \in H_{0}(\operatorname{curl}, \Omega) \\
& (\nabla q, \mathbf{u}(t))=0 \quad \forall q \in H_{0}^{1}(\Omega) \\
& \mathbf{u}(0)=0
\end{aligned}
$$

and (Problem 2$)$ has the following week variational formulation :

$$
\begin{aligned}
& \text { Find } \mathbf{u}(t) \in H(\mathbf{c u r l}, \Omega) \text { and } p(t) \in H^{1}(\Omega) / \mathbb{R} \text { such that } \\
& \left(\frac{d}{d t} \mathbf{u}(t), \mathbf{v}\right)+\nu(\operatorname{curl} \mathbf{u}(t), \mathbf{c u r l} \mathbf{v})+(\nabla p(t), \mathbf{v})=(\mathbf{f}(t), \mathbf{v}) \quad \forall \mathbf{v} \in H(\operatorname{curl}, \Omega) \\
& (\nabla q, \mathbf{u}(t))=0 \quad \forall q \in H^{1}(\Omega) \\
& \mathbf{u}(0)=0
\end{aligned}
$$

Where $\mathbf{u}(t)=\mathbf{u}(t,$.$) and p(t)=p(t,$.$) .$

Each variational formulation is splitted into a system for the velocity and a Poisson equation for the pressure.

Let us introduce the spaces :

$$
V_{0}=\left\{\mathbf{v} \in H_{0}(\mathbf{c u r l}, \Omega) ;(\nabla q, \mathbf{v})=0 \forall q \in H_{0}^{1}(\Omega)\right\}
$$

and

$$
U=\left\{\mathbf{v} \in H(\mathbf{c u r l}, \Omega) ;(\nabla q, \mathbf{v})=0 \forall q \in H^{1}(\Omega)\right\} .
$$

The lemma (2.1) and the theorems (2.2) and (2.3) allow us to establish the following theorem: 
Theorem 2.4. The problem (2.2)-(2.3) is equivalent to the problem :

Find $\mathbf{u}(t) \in V_{0}$ such that

$$
\left(\frac{d}{d t} \mathbf{u}(t), \mathbf{v}\right)+\nu(\operatorname{curl} \mathbf{u}(t), \operatorname{curl} \mathbf{v})=(\mathbf{f}(t), \mathbf{v}) \quad \forall \mathbf{v} \in V_{0}
$$

Find $p(t) \in H_{0}^{1}(\Omega)$ such that

$$
(\nabla p(t), \nabla q)=(\mathbf{f}(t), \nabla q) \quad \forall q \in H_{0}^{1}(\Omega) .
$$

The problem (2.5)-(2.6) is equivalent to the problem:

Find $\mathbf{u}(t) \in U$ such that

$$
\left(\frac{d}{d t} \mathbf{u}(t), \mathbf{v}\right)+\nu(\operatorname{curl} \mathbf{u}(t), \operatorname{curl} \mathbf{v})=(\mathbf{f}(t), \mathbf{v}) \quad \forall \mathbf{v} \in U
$$

Find $p(t) \in H^{1}(\Omega) / \mathbb{R}$ such that

$$
(\nabla p(t), \nabla q)=(\mathbf{f}(t), \nabla q) \quad \forall q \in H^{1}(\Omega) .
$$

Furthermore, the first problem has one and only one solution $(\mathbf{u}, p) \in\left(L^{\infty}\left(0, T ; L^{2}(\Omega)^{3}\right) \cap L^{2}\left(0, T ; V_{0}\right) \times\right.$ $\left.L^{\infty}\left(0, T ; H_{0}^{1}(\Omega)\right)\right)$ and the second problem has one and only one solution $(\mathbf{u}, p) \in\left(L^{\infty}\left(0, T ; L^{2}(\Omega)^{3}\right) \cap\right.$ $\left.L^{2}(0, T ; U) \times L^{\infty}\left(0, T ; H^{1}(\Omega) / \mathbb{R}\right)\right)$. In both cases, we have the following bounds :

$$
\begin{gathered}
\|p\|_{L^{\infty}\left(0, T ; H_{0}^{1}(\Omega)\right)} \leq\|\mathbf{f}\|_{L^{\infty}\left(0, T ; L^{2}(\Omega)^{3}\right)} \\
\|\mathbf{u}\|_{L^{\infty}\left(0, T ; L^{2}(\Omega)^{3}\right)}+\|\mathbf{c u r l} \mathbf{u}\|_{L^{2}\left(0, T ; L^{2}(\Omega)^{3}\right)} \leq C_{1}\|\mathbf{f}\|_{L^{\infty}\left(0, T ; L^{2}(\Omega)^{3}\right)}
\end{gathered}
$$

\section{Finite Element Discretization}

We introduce a regular family of tetrahedra $\left(\tau_{h}\right)_{h}$ in the sens that :

- for each $h, \bar{\Omega}$ in the union of all element of $\tau_{h}$;

- for each $h$, the intersection of two different elements of $\tau_{h}$, if not empty, is a corner, a whole edge or a whole face of both of them;

- the ratio of the diameter $h_{\kappa}$ of an element $\kappa$ in $\tau_{h}$ to the diameter of its inscribed sphere is bounded by a constant independent of $\kappa$ and $h$;

As usual, $h$ denotes the maximum of the diameters of the elements of $\tau_{h}$.

Next, for each $\kappa$ in $\tau_{h}$, we introduce the spaces $\mathbb{P}_{0}(\kappa)$ of the restrictions to $\kappa$ of constant functions on $\mathbb{R}^{3}, \mathbb{P}_{1}(\kappa)$ of the restrictions to $\kappa$ of affine functions on $\mathbb{R}$ and the space $\mathbb{P}_{K}(\kappa)$ of the restrictions to $\kappa$ of polynomials $\mathbf{v}$ of the form :

$$
\mathbf{v}(x)=a+b \times x, \quad a \in \mathbb{R}^{3}, b \in \mathbb{R}^{3} .
$$

The space $\mathbb{P}_{K}(\kappa)$ and the corresponding finite elements are studied in [20].

Their degrees of freedom are the average flux along the edges $\int_{l}(\mathbf{v} . \mathbf{t}) d l$, for the six edges $l$ of $\kappa, t$ is the direction vector of $l$.

Hence, we associate the operator $r_{\kappa}$ where $r_{\kappa}(\mathbf{u})$ is the unique polynomial of $\mathbb{P}_{K}$ that has the same flux along the edges as $\mathbf{u}$. We define also the the operator $I_{\kappa}$ where $I_{\kappa}(q)$ is the unique polynomial of $\mathbb{P}_{1}(\kappa)$ that has the same values on the vertex of $\kappa$ as $q$.

Next, let us introduce the discrete spaces :

$$
\begin{aligned}
M_{h} & =\left\{\mathbf{u}_{h} \in H(\mathbf{c u r l}, \Omega) ;\left.\mathbf{u}_{h}\right|_{\kappa} \in \mathbb{P}_{K}(\kappa), \forall \kappa \in \tau_{h}\right\} \\
M_{0 h} & =M_{h} \cap H_{0}(\operatorname{curl}, \Omega), \\
Q_{h} & =\left\{q_{h} \in C^{0}(\bar{\Omega}) ;\left.q_{h}\right|_{\kappa} \in \mathbb{P}_{1}(\kappa), \forall \kappa \in \tau_{h}\right\} \\
Q_{0 h} & =Q_{h} \cap H_{0}^{1}(\Omega)
\end{aligned}
$$


With these spaces, the finite dimensional analogues of $V_{0}$ and $U$ are :

$$
\begin{aligned}
& V_{0 h}=\left\{\mathbf{v}_{h} \in M_{0 h} ;\left(\nabla q_{h}, \mathbf{v}_{h}\right)=0, \quad \forall q_{h} \in Q_{h}\right\} \\
& \text { and } \\
& U_{h}=\left\{\mathbf{v}_{h} \in M_{h} ;\left(\nabla q_{h}, \mathbf{v}_{h}\right)=0 \quad \forall q_{h} \in Q_{h}\right\} .
\end{aligned}
$$

We define the interpolation operators $r_{h}$ from $H^{1}(\Omega)^{3}$ onto $M_{h}, I_{h}$ from $H^{2}(\Omega)$ onto $Q_{h}$ by

$$
\left.r_{h} u=r_{\kappa}(u) \text { on } \kappa, \forall \kappa \in \tau_{h} \quad \text { (similarly for } I_{h}\right) \text {. }
$$

Theorem 3.1. Assume that the triangulation $\tau_{h}$ is regular. For all $k \geq 1$ we have :

$$
\left\|\mathbf{u}-r_{h} \mathbf{u}\right\|_{0, \Omega}+h\left\|\mathbf{c u r l}\left(\mathbf{u}-r_{h} \mathbf{u}\right)\right\|_{0, \Omega} \leq C h|\mathbf{u}|_{1, t, \Omega}, \forall \mathbf{u} \in W^{1, t}(\Omega)^{3}, \text { for some } t>2 .
$$

Moreover, when $\mathbf{u} \in\left(H^{k}(\Omega)\right)^{3}$ and $\forall k \geq 2$ we have :

$$
\left\|\mathbf{u}-r_{h} \mathbf{u}\right\|_{0, \Omega} \leq C h^{k}|\mathbf{u}|_{k, \Omega}
$$

and, when $\mathbf{u} \in\left(H^{k+1}(\Omega)\right)^{3}$ and $\forall k \geq 1$ we have :

$$
\left\|\operatorname{curl}\left(\mathbf{u}-r_{h} \mathbf{u}\right)\right\|_{0, \Omega} \leq C h^{k}|\mathbf{u}|_{k+1, \Omega}
$$

We have also an important result given by V. Girault [16] which shows an impact imbedding between the spaces $V_{0 h}$ or $U_{h}$ and $L^{4}(\Omega)^{3}$.

Theorem 3.2. Let $\tau_{h}$ a uniformly regular triangulation of $\Omega$. For each space $V_{0 h}$ and $U_{h}$, there exists constants $C$ and $c^{\prime}$, independent of $h$, such that

$$
\left\|\mathbf{u}_{h}\right\|_{2, \Omega} \leq C|| \mathbf{u}_{h}\left\|_{0,4, \Omega} \leq C^{\prime}\right\| \mathbf{c u r l} \mathbf{u}_{h} \|_{0, \Omega} \quad \forall \mathbf{u}_{h} \in V_{0 h} \text { or } U_{h}
$$

To discretize in time, we divide the interval $[0, T]$ into $N$ subintervals of equal length $k=\frac{T}{N}$, with grid-points $t^{n}=n k, 0 \leq n \leq N$.

We discretize (Problem 1$)$ by : knowing $u_{h}^{n}$, find $\left(u_{h}^{n+1}, p_{h}^{n+1}\right)$ with values in $V_{0 h} \times Q_{0 h}$, solution of

$$
\frac{1}{k}\left(\mathbf{u}_{h}^{n+1}-\mathbf{u}_{h}^{n}, \mathbf{v}_{h}\right)+\nu\left(\operatorname{curl} \mathbf{u}_{h}^{n+1}, \operatorname{curl} \mathbf{v}_{h}\right)+\left(\nabla p_{h}^{n+1}, \mathbf{v}_{h}\right)=\left(\mathbf{f}^{n+1}, \mathbf{v}_{h}\right) \quad \forall \mathbf{v}_{h} \in M_{0 h}
$$

Similarly, we discretize (Problem 2$)$ by : knowing $u_{h}^{n}$, find $\left(u_{h}^{n+1}, p_{h}^{n+1}\right)$ with values in $U_{h} \times Q_{h} / \mathbb{R}$, solution of

$$
\frac{1}{k}\left(\mathbf{u}_{h}^{n+1}-\mathbf{u}_{h}^{n}, \mathbf{v}_{h}\right)+\nu\left(\mathbf{c u r} \mathbf{u}_{h}^{n+1}, \mathbf{c u r l} \mathbf{v}_{h}\right)+\left(\nabla p_{h}^{n+1}, \mathbf{v}_{h}\right)=\left(\mathbf{f}^{n+1}, \mathbf{v}_{h}\right) \quad \forall \mathbf{v}_{h} \in M_{h} .
$$

Where we assume that $\mathbf{f}^{n+1}=\mathbf{f}\left(t_{n+1}\right]$ and $\mathbf{u}_{h}^{0}=\mathbf{0}$.

As in the continuous way, the problem (3.6) can be splited to

Find $\mathbf{u}_{h}^{n+1} \in V_{0 h}$ such that

$$
\frac{1}{k}\left(\mathbf{u}_{h}^{n+1}-\mathbf{u}_{h}^{n}, \mathbf{v}_{h}\right)+\nu\left(\operatorname{curl} \mathbf{u}_{h}^{n+1}, \operatorname{curl} \mathbf{v}_{h}\right)=\left(\mathbf{f}^{n+1}, \mathbf{v}_{h}\right) \quad \forall \mathbf{v}_{h} \in V_{0 h},
$$

Find $p_{h}^{n+1} \in Q_{0 h}$ such that

$$
\left(\nabla p_{h}^{n+1}, \nabla q_{h}\right)=\left(\mathbf{f}^{n+1}, \nabla q_{h}\right), \quad \forall q_{h} \in Q_{0 h} .
$$

And the problem (3.7) can be splited to 
Find $\mathbf{u}_{h}^{n+1} \in U_{h}$ such that

$$
\frac{1}{k}\left(\mathbf{u}_{h}^{n+1}-\mathbf{u}_{h}^{n}, \mathbf{v}_{h}\right)+\nu\left(\operatorname{curl} \mathbf{u}_{h}^{n+1}, \operatorname{curl} \mathbf{v}_{h}\right)=\left(\mathbf{f}^{n+1}, \mathbf{v}_{h}\right), \quad \forall \mathbf{v}_{h} \in U_{h},
$$

Find $p_{h}^{n+1} \in Q_{h} / \mathbb{R}$ such that

$$
\left(\nabla p_{h}^{n+1}, \nabla q_{h}\right)=\left(\mathbf{f}^{n+1}, \nabla q_{h}\right), \quad \forall q_{h} \in Q_{h} .
$$

It is easy to show that these two last discrete problems have a unique solution.

The pressure is entirely dissociated from the velocity, i.e. can be computed without knowing the velocity. We have also for both discrete problems :

$$
\sup _{0 \leq n \leq N-1}\left\|\mathbf{u}_{h}^{n+1}\right\|_{0, \Omega}^{2}+\sum_{n=0}^{N-1}\left\|\mathbf{u}_{h}^{n+1}-\mathbf{u}_{h}^{n}\right\|_{0, \Omega}^{2}+\sum_{n=0}^{N-1} k\left\|\mathbf{c u r l} \mathbf{u}_{h}^{n+1}\right\|_{0, \Omega}^{2} \leq C\|\mathbf{f}\|_{L^{\infty}\left(0, T ; L^{2}(\Omega)^{3}\right)}^{2}
$$

and

$$
\sup _{0 \leq n \leq N-1}\left|p_{h}^{n+1}\right|_{1, \Omega} \leq\|\mathbf{f}\|_{L^{\infty}\left(0, T ; L^{2}(\Omega)^{3}\right)}
$$

\section{A PRIORI ERROR ANALYSIS}

In this section, we will establish the a priori error estimates for the pressure and the velocity.

Theorem 4.1. We suppose that the theoretical solution $(\mathbf{u}, p)$ of the problem (2.8)-(2.9) (resp. (2.10)(2.11)) verify: $\mathbf{u} \in L^{\infty}\left(0, T ; H^{2}(\Omega)^{3}\right), \mathbf{u}^{\prime} \in L^{2}\left(0, T ; H^{1}(\Omega)^{3}\right), \mathbf{u}^{\prime \prime} \in L^{\infty}\left(0, T ; L^{2}(\Omega)^{3}\right)$ and $p \in L^{\infty}\left(0, T ; H^{2}(\Omega)\right)$. Then they verify with the numerical solution $\left(\mathbf{u}_{h}^{n+1}, p_{h}^{n+1}\right)$ of the problem (3.8)-(3.9) (resp. (3.10)-(3.11)) the error estimates :

$$
\begin{aligned}
\sup _{0 \leq n \leq N-1} & \left\|\nabla\left(p\left(t_{n+1}\right)-p_{h}^{n+1}\right)\right\|_{0, \Omega} \leq C(p, T, \Omega) h \\
\sup _{0 \leq n \leq N-1}\left\|\mathbf{u}\left(t_{n+1}\right)-\mathbf{u}_{h}^{n+1}\right\|_{0, \Omega}^{2}+ & \sum_{n=0}^{N-1}\left\|\left(\mathbf{u}\left(t_{n+1}\right)-\mathbf{u}_{h}^{n+1}\right)-\left(\mathbf{u}\left(t_{n}\right)-\mathbf{u}_{h}^{n}\right)\right\|_{0, \Omega}^{2} \\
& +\sum_{n=0}^{N-1} k\left\|\mathbf{c u r l}\left(\mathbf{u}\left(t_{n+1}\right)-\mathbf{u}_{h}^{n+1}\right)\right\|_{0, \Omega}^{2} \leq C(p, \mathbf{u}, \mathbf{f}, T, \Omega)\left(h^{2}+k^{2}\right)
\end{aligned}
$$

Proof:

For the pressure, let us choose $q=q_{h}$ in (2.9) and take, for $t=t_{n+1}$, the difference with (3.9) (resp. (2.11) and (3.11)), we obtain:

$$
\left(\nabla\left(p\left(t_{n+1}\right)-p_{h}^{n+1}\right), \nabla q_{h}\right)=0, \quad \forall q_{h} \in Q_{0 h} \quad\left(\text { resp. } Q_{h}\right)
$$

then $\forall q_{h} \in Q_{0 h} \quad\left(\right.$ resp. $\left.Q_{h}\right)$

$$
\left(\nabla\left(I_{h} p\left(t_{n+1}\right)-p_{h}^{n+1}\right), \nabla q_{h}\right)=-\left(\nabla\left(p\left(t_{n+1}\right)-I_{h} p\left(t_{n+1}\right)\right), \nabla q_{h}\right)
$$

We take $q_{h}=I_{h} p\left(t_{n+1}\right)-p_{h}^{n+1}$ and we obtain, using the triangle inequality, the relation:

$$
\|\left.\nabla\left(p\left(t_{n+1}\right)-p_{h}^{n+1}\right)\right|_{0, \Omega} \leq C h\left|p\left(t_{n+1}\right)\right|_{2, \Omega}
$$

which lead to the first result (4.1).

For the velocity, we choose $\mathbf{v}=\mathbf{v}_{h}$ in the equation (2.8) taken for $t=t_{n+1}$ and multiplied by $k$, and take the difference with (3.8) multiplied by $k$ (resp. (2.10) and (3.10)), we obtain:

$$
\begin{aligned}
\left(k \frac{d \mathbf{u}}{d t}\left(t_{n+1}\right)-\left(\mathbf{u}_{h}^{n+1}-\mathbf{u}_{h}^{n}\right), \mathbf{v}_{h}\right)+ & \nu k\left(\operatorname{curl}\left(\mathbf{u}\left(t_{n+1}\right)-\mathbf{u}_{h}^{n+1}\right), \operatorname{curl} \mathbf{v}_{h}\right) \\
& +k\left(\nabla\left(p\left(t_{n+1}\right)-p_{h}^{n+1}\right), \mathbf{v}_{h}\right)=0 \quad \forall \mathbf{v}_{h} \in M_{0 h} \quad\left(\text { resp. } M_{h}\right)
\end{aligned}
$$


Then a simple manipulation gives:

$$
\begin{aligned}
& \left(\left(r_{h} \mathbf{u}\left(t_{n+1}\right)-\mathbf{u}_{h}^{n+1}\right)-\left(r_{h} \mathbf{u}\left(t_{n}\right)-\mathbf{u}_{h}^{n}\right), \mathbf{v}_{h}\right)+\nu k\left(\operatorname{curl}\left(r_{h} \mathbf{u}\left(t_{n+1}\right)-\mathbf{u}_{h}^{n+1}\right), \operatorname{curl} \mathbf{v}_{h}\right)= \\
& -\left(\left(\mathbf{u}\left(t_{n+1}\right)-r_{h} \mathbf{u}\left(t_{n+1}\right)\right)-\left(\mathbf{u}\left(t_{n}\right)-r_{h} \mathbf{u}\left(t_{n}\right)\right), \mathbf{v}_{h}\right)+\left(\left(\mathbf{u}\left(t_{n+1}\right)-\mathbf{u}\left(t_{n}\right)\right)-k \mathbf{u}^{\prime}\left(t_{n+1}\right), \mathbf{v}_{h}\right) \\
& -\nu k\left(\operatorname{curl}\left(\mathbf{u}\left(t_{n+1}\right)-r_{h} \mathbf{u}\left(t_{n+1}\right)\right), \operatorname{curl} \mathbf{v}_{h}\right)-k\left(\nabla\left(p\left(t_{n+1}\right)-p_{h}^{n+1}\right), \mathbf{v}_{h}\right)
\end{aligned}
$$$$
\forall \mathbf{v}_{h} \in M_{0 h} \quad\left(\operatorname{resp} . M_{h}\right)
$$

We take $\mathbf{v}_{h}=\mathbf{v}_{h}^{n+1}=r_{h} \mathbf{u}\left(t_{n+1}\right)-\mathbf{u}_{h}^{n+1}$ and use the formula $(a-b, a)=\frac{1}{2} a^{2}-\frac{1}{2} b^{2}+\frac{1}{2}(a-b)^{2}$, we obtain :

$$
\begin{aligned}
& \frac{1}{2}\left\|\mathbf{v}_{h}^{n+1}\right\|_{0, \Omega}^{2}-\frac{1}{2}\left\|\mathbf{v}_{h}^{n}\right\|_{0, \Omega}^{2}+\frac{1}{2}\left\|\left(\mathbf{v}_{h}^{n+1}-\mathbf{v}_{h}^{n}\right)\right\|_{0, \Omega}^{2}+\nu k\left\|\operatorname{curl}\left(\mathbf{v}_{h}^{n+1}\right)\right\|_{0, \Omega}^{2} \leq \\
& \left|\left(\int_{t_{n}}^{t_{n+1}}\left(\mathbf{u}^{\prime}(t)-r_{h} \mathbf{u}^{\prime}(t)\right) d t, \mathbf{v}_{h}^{n+1}\right)\right|+C_{2} k^{2}\left\|\mathbf{u}^{\prime \prime}\right\|_{L^{\infty}\left(0, T ; L^{2}(\Omega)^{3}\right)}\left\|\mathbf{v}_{h}^{n+1}\right\|_{0, \Omega} \\
& +\nu C_{3} k h\left\|\mathbf{u}\left(t_{n+1}\right)\right\|_{2, \Omega}\left\|\mathbf{c u r l} \mathbf{v}_{h}^{n+1}\right\|_{0, \Omega}+\left|k\left(\nabla\left(p\left(t_{n+1}\right)-p_{h}^{n+1}\right), \mathbf{v}_{h}^{n+1}\right)\right|
\end{aligned}
$$

The first term of the right member can be treated as :

$$
\left|\left(\int_{t_{n}}^{t_{n+1}}\left(\mathbf{u}^{\prime}(t)-r_{h} \mathbf{u}^{\prime}(t)\right) d t, \mathbf{v}_{h}^{n+1}\right)\right| \leq C_{1}\left(\frac{1}{2 \varepsilon_{1}} h^{2}\left\|\mathbf{u}^{\prime}\right\|_{L^{2}\left(t_{n}, t_{n+1} ; H_{0}^{1}(\Omega)^{3}\right)}^{2}+\frac{\varepsilon_{1}}{2} k\left\|\mathbf{v}_{h}^{n+1}\right\|_{0, \Omega}^{2}\right)
$$

The second term :

$$
\left|C_{2} k^{2}\left\|\mathbf{u}^{\prime \prime}\right\|_{L^{\infty}\left(0, T ; L^{2}(\Omega)^{3}\right)}\right|\left|\mathbf{v}_{h}^{n+1} \|_{0, \Omega}\right| \leq C_{2}\left\{\frac{1}{2 \varepsilon_{2}} k^{3}\left\|\mathbf{u}^{\prime \prime}\right\|_{L^{\infty}\left(0, T ; L^{2}(\Omega)^{3}\right)}^{2}+\frac{\varepsilon_{2}}{2} k\left\|\mathbf{v}_{h}^{n+1}\right\|_{0, \Omega}^{2}\right\}
$$

The third term :

$$
\left|\nu C_{3} k h\left\|\mathbf{u}\left(t_{n+1}\right)\right\|_{2, \Omega}\right|\left|\mathbf{c u r l} \mathbf{v}_{h}^{n+1} \|_{0, \Omega}\right| \leq C_{3}\left\{\frac{\nu^{2}}{2 \varepsilon_{3}} k h^{2}\|\mathbf{u}\|_{L^{\infty}\left(0, T ; H^{2}(\Omega)^{3}\right)}^{2}+\frac{\varepsilon_{3}}{2} k\left\|\mathbf{c u r l} \mathbf{v}_{h}^{n+1}\right\|_{0, \Omega}^{2}\right\}
$$

The last term: for all functions $\mathbf{v}_{h}^{n+1}$ of $M_{0 h}\left(\operatorname{resp} M_{h}\right.$ ), we define $q_{h}^{n+1}$ in $Q_{0 h}$ (resp. $Q_{h}$ ) by

$$
\left(\nabla q_{h}^{n+1}, \nabla \mu_{h}\right)=\left(\mathbf{v}_{h}^{n+1}, \nabla \mu_{h}\right) \quad \forall \mu_{h} \in Q_{0 h} \quad\left(\text { resp. } Q_{h}\right)
$$

and set $\mathbf{w}_{h}^{n+1}=\mathbf{v}_{h}^{n+1}-\nabla q_{h}^{n+1}$. Then $\mathbf{w}_{h}^{n+1}$ belongs to $V_{0 h}\left(\right.$ resp. $\left.U_{h}\right)$ and $\operatorname{curl} \mathbf{w}_{h}^{n+1}=\mathbf{c u r l} \mathbf{v}_{h}^{n+1}$. Then we obtain with the relation (4.3) :

$$
\begin{aligned}
\left|k\left(\nabla\left(p\left(t_{n+1}\right)-p_{h}^{n+1}\right), \mathbf{v}_{h}^{n+1}\right)\right| & =\left|k\left(\nabla\left(p\left(t_{n+1}\right)-p_{h}^{n+1}\right), \mathbf{w}_{h}^{n+1}\right)\right| \\
& \leq C_{4}\left(\frac{1}{2 \varepsilon_{4}} k\left\|\nabla\left(p\left(t_{n+1}\right)-p_{h}^{n+1}\right)\right\|_{0, \Omega}^{2}+\frac{\varepsilon_{4}}{2} k\left\|\mathbf{c u r l} \mathbf{v}_{h}^{n+1}\right\|_{0, \Omega}^{2}\right)
\end{aligned}
$$

Taking all the last inequality, summing over $n$ from 0 to $m-1$, we obtain by choosing $\varepsilon_{1}=\frac{1}{8 C_{1} T} ; \varepsilon_{2}=$ $\frac{1}{8 C_{2} T}, \varepsilon_{3}=\frac{\nu}{2 C_{3}}, \varepsilon_{4}=\frac{\nu}{2 C_{4}}:$

$\frac{1}{2}\left\|\mathbf{v}_{h}^{m}\right\|_{0, \Omega}^{2}+\frac{1}{2} \sum_{n=0}^{m-1}\left\|\left(\mathbf{v}_{h}^{n+1}-\mathbf{v}_{h}^{n}\right)\right\|_{0, \Omega}^{2}+\frac{\nu}{2} \sum_{n=0}^{m-1} k\left\|\operatorname{curl}\left(\mathbf{v}_{h}^{n+1}\right)\right\|_{0, \Omega}^{2} \leq C\left(h^{2}+k^{2}\right)+\frac{1}{8} \sup _{1 \leq n \leq N-1}\left\|\mathbf{v}_{h}^{n+1}\right\|_{0, \Omega}^{2}$

which leads to :

$$
\frac{3}{8} \sup _{1 \leq n \leq N-1}\left\|\mathbf{v}_{h}^{n+1}\right\|_{0, \Omega}^{2}+\frac{1}{2} \sum_{n=0}^{N-1}\left\|\left(\mathbf{v}_{h}^{n+1}-\mathbf{v}_{h}^{n}\right)\right\|_{0, \Omega}^{2}+\frac{\nu}{2} \sum_{n=0}^{N-1} k\left\|\operatorname{curl}\left(\mathbf{v}_{h}^{n+1}\right)\right\|_{0, \Omega}^{2} \leq C^{\prime}\left(h^{2}+k^{2}\right)
$$

We obtain the result by using the triangular inequality:

$$
\left\|\mathbf{u}\left(t_{n+1}\right)-\mathbf{u}_{h}^{n+1}\right\|_{0, \Omega} \leq\left\|\mathbf{u}\left(t_{n+1}\right)-r_{h} \mathbf{u}\left(t_{n+1}\right)\right\|_{0, \Omega}+\left\|r_{h} \mathbf{u}\left(t_{n+1}\right)-\mathbf{u}_{h}^{n+1}\right\|_{0, \Omega}
$$


and remarking that:

$$
\begin{aligned}
\left\|\left(\mathbf{u}\left(t_{n+1}\right)-\mathbf{u}_{h}^{n+1}\right)-\left(\mathbf{u}\left(t_{n}\right)-\mathbf{u}_{h}^{n}\right)\right\|_{0, \Omega}^{2} & \leq 2\left\|\int_{t_{n}}^{t_{n+1}}\left(\mathbf{u}^{\prime}(\tau)-r_{h} \mathbf{u}^{\prime}(\tau)\right) d \tau\right\|_{0, \Omega}^{2}+2\left\|\mathbf{v}_{h}^{n+1}-\mathbf{v}_{h}^{n}\right\|_{0, \Omega}^{2} \\
& \leq C k h^{2}\left\|\nabla \mathbf{u}^{\prime}\right\|_{L^{2}\left(t_{n}, t_{n+1} ; L^{2}(\Omega)^{3}\right)}^{2}+2\left\|\mathbf{v}_{h}^{n+1}-\mathbf{v}_{h}^{n}\right\|_{0, \Omega}^{2}
\end{aligned}
$$

Remark: We denote by $\mathbf{u}_{h}$ and $p_{h}$ the time dependent functions which take in the interval $\left[t_{n}, t_{n+1}\right]$ the values:

$$
\begin{aligned}
& \mathbf{u}_{h}(t)=\frac{t-t_{n}}{k}\left(\mathbf{u}_{h}^{n+1}-\mathbf{u}_{h}^{n}\right)+\mathbf{u}_{h}^{n} \\
& p_{h}(t)=\frac{t-t_{n}}{k}\left(p_{h}^{n+1}-p_{h}^{n}\right)+p_{h}^{n}
\end{aligned}
$$

We have, with some regularities over $(\mathbf{u}, p)$, the same a priori estimates as the last theorem:

$$
\begin{gathered}
\left\|\nabla\left(p-p_{h}\right)\right\|_{L^{\infty}\left(0, T ; L^{2}(\Omega)\right)}^{2} \leq C\left(h^{2}+k^{2}\right) \\
\left\|\mathbf{u}-\mathbf{u}_{h}\right\|_{L^{\infty}\left(0, T ; L^{2}(\Omega)^{3}\right)}^{2}+\nu\left\|\operatorname{curl}\left(\mathbf{u}-\mathbf{u}_{h}\right)\right\|_{L^{2}\left(0, T ; L^{2}(\Omega)^{3}\right)}^{2} \leq C\left(h^{2}+k^{2}\right)
\end{gathered}
$$

If fact, it suffices to use the inequality:

$$
\left|\nabla\left(p(t)-p_{h}(t)\right)\right| \leq\left|\nabla\left(p(t)-p\left(t_{n+1}\right)\right)\right|+\left|\nabla\left(p\left(t_{n+1}\right)-p_{h}^{n+1}\right)\right|+\left|\nabla\left(p_{h}^{n+1}-p_{h}(t)\right)\right|
$$

and remark that:

$$
\left|\nabla\left(p_{h}^{n+1}-p_{h}(t)\right)\right| \leq\left|\nabla\left(p_{h}^{n+1}-p_{h}^{n}\right)\right| \leq\left|\nabla\left(p_{h}^{n+1}-p\left(t_{n+1}\right)\right)\right|+\left|\nabla\left(p\left(t_{n+1}\right)-p\left(t_{n}\right)\right)\right|+\left|\nabla\left(p\left(t_{n}\right)-p_{h}^{n}\right)\right|
$$

\section{A POSTERIORI ERROR ANALYSIS}

We now intend to prove a posteriori error estimates between the exact solution $(\mathbf{u}, p)$ of the problem (2.8)-(2.9) and the numerical solution $\left(\mathbf{u}_{h}, p_{h}\right)$ of the problem (3.8)-(3.9). By the same way, we can prove a posteriori error estimates between the solution $(\mathbf{u}, p)$ of the exact problem $(2.10)-(2.11)$ and $\left(\mathbf{u}_{h}, p_{h}\right)$ of the numerical problem (3.10)-(3.11). In all the rest of the paper, we suppose that $\mathbf{f} \in L^{\infty}(0, T ; H(\operatorname{div}, \Omega))$.

We first introduce the space

$$
Z_{h}=\left\{\mathbf{g}_{h} \in L^{2}(\Omega)^{3} ; \forall \kappa \in \tau_{h},\left.\mathbf{g}_{h}\right|_{\kappa} \in \mathbb{P}_{0}(\kappa)\right\}
$$

and we fix an approximation $\rho_{h}(\mathbf{f})$ of the data $\mathbf{f}$ in $Z_{h}$.

Next, we denote by $\varepsilon_{h}$ the set of all faces of the elements of $\tau_{h}$ that are not contained in $\Gamma$. For every element $\kappa$ in $\tau_{h}$, we denote by $\varepsilon_{\kappa}$ the set of faces of $\kappa$ that are not contained in $\Gamma, \Delta_{\kappa}$ the set of union of elements of $\tau_{h}$ that intersect $\kappa, \Delta_{e}$ the union of elements of $\tau_{h}$ that intersect the face $e, h_{\kappa}$ the diameter of $\kappa$ and $h_{e}$ the diameter of the face $e$. Also, $\mathbf{n}_{\kappa}$ stands for the unit outward normal vector to $\kappa$ on $\partial \kappa$ and $[\cdot]_{e}$ the jump through the face $e$ of $\kappa$.

For the demonstration of the next theorems, we introduce for an element $\kappa$ of $\tau_{h}$, the bull function $\psi_{\kappa}$ (resp. $\psi_{e}$ of the face $e$ ) which is equal to the product of the $d+1$ barycentric coordinates associated with the vertices of $\kappa$ (resp. of $e$ ) and $\mathcal{L}_{e}$ the lifting operator from polynomials defined on $e$ into polynomials defined on the elements $\kappa$ and $\kappa^{\prime}$ contained $e$, which is constructed by affine transformations from a fixed operator on the reference element.

Property 5.1. Denoting by $P_{r}(\kappa)$ the polynomial of degrees $r$ on $\kappa$, we have

$$
\forall v \text { polynom of } P_{r}(\kappa) \quad\left\{\begin{array}{l}
c\|v\|_{0, \kappa} \leq\left\|v \psi_{\kappa}^{1 / 2}\right\|_{0, \kappa} \leq c^{\prime}\|v\|_{0, \kappa} \\
|v|_{1, \kappa} \leq c h_{k}^{-1}\|v\|_{0, \kappa}
\end{array}\right.
$$


Property 5.2. Denoting by $P_{r}(e)$ the polynomial of degrees $r$ on $e$, we have

$$
\forall v \text { polynom of } P_{r}(e) \quad c\|v\|_{0, e} \leq\left\|v \psi_{e}^{1 / 2}\right\|_{0, e} \leq c^{\prime}\|v\|_{0, e}
$$

and $\forall v$ polynom of $P_{r}(e)$ which vanishes on $\partial e, \kappa$ is a element which contains $e$, we have

$$
\left\|\mathcal{L}_{e} v\right\|_{0, \kappa}+h_{e}\left|\mathcal{L}_{e} v\right|_{1, \kappa} \leq \operatorname{ch}_{e}^{1 / 2}\|v\|_{0, e} .
$$

We denote by $R_{h}$ the Clément operator [10]. We have for all function $q \in H_{0}^{1}(\Omega), R_{h} q \in Q_{0 h}$ verifies

$$
\begin{aligned}
& \left\|q-R_{h} q\right\|_{0, \kappa} \leq c h_{\kappa}\|q\|_{1, \Delta \kappa} \\
& \left\|q-R_{h} q\right\|_{0, e} \leq c h_{e}^{1 / 2}\|q\|_{1, \Delta e}
\end{aligned}
$$

Furtheremore, we denote by $\mathcal{R}_{h}$ the Raviart-Thomas operator: for any smooth enough vectorial function $\mathbf{v}, \mathcal{R}_{h} \mathbf{v}$ belongs to $M_{0 h}$ and satisfies

$$
\forall e \in \varepsilon_{h}, \quad \int_{e}\left(\mathbf{v}-\mathcal{R}_{\mathbf{h}} \mathbf{v}\right) \cdot \mathbf{n} d \tau=0 .
$$

Moreover, this operator satisfies, see [24]: $\forall \mathbf{v}$ in $H^{1}(\Omega)^{3}$ and $\forall \kappa$ in $\tau_{h}$,

$$
\begin{aligned}
& \left\|\mathbf{v}-\mathcal{R}_{h} \mathbf{v}\right\|_{0, \kappa} \leq c h_{\kappa}\|\mathbf{v}\|_{1, \kappa} \\
& \left\|\mathbf{v}-\mathcal{R}_{h} \mathbf{v}\right\|_{0, e} \leq c h_{e}^{1 / 2}\|\mathbf{v}\|_{1, \Delta e}
\end{aligned}
$$

Let us begin with a posteriori error for the pressure. We introduce the function:

$$
\mathbf{f}^{k}(t)=\frac{t-t_{n}}{k}\left(\mathbf{f}\left(t_{n+1}\right)-\mathbf{f}\left(t_{n}\right)\right)+\mathbf{f}\left(t_{n}\right) \quad \forall t \in\left[t_{n}, t_{n+1}\right]
$$

and we deduce that:

$$
\left(\nabla p_{h}(t)-\mathbf{f}^{k}(t), \nabla q_{h}(t)\right)=0
$$

So, we have:

$\forall q(t) \in H_{0}^{1}(\Omega) \quad\left(\operatorname{resp} . H^{1}(\Omega) / \mathbb{R}\right)$

$\left(\nabla\left(p(t)-p_{h}(t)\right), \nabla q(t)\right)=\left(\mathbf{f}(t)-\nabla p_{h}(t), \nabla q(t)\right)=\left(\mathbf{f}(t)-\mathbf{f}^{k}(t), \nabla q(t)\right)+\left(\mathbf{f}^{k}(t)-\nabla p_{h}(t), \nabla\left(q(t)-q_{h}(t)\right)\right)$

We define the error indicator by

$$
\eta_{n, \kappa}^{2}=\sum_{e \in \varepsilon_{\kappa}} h_{e}\left\|\left[\left(\rho_{h} \mathbf{f}^{k}-\nabla p_{h}\right) \cdot \mathbf{n}\right]\right\|_{L^{2}\left(t_{n}, t_{n+1} ; L^{2}(e)\right)}^{2}
$$

Lemma 5.3. The following estimate hold

$$
\begin{aligned}
& \left\|\nabla\left(p-p_{h}\right)\right\|_{L^{2}\left(0, T ; L^{2}(\Omega)\right)}^{2} \leq C\left\{\sum _ { n = 0 } ^ { N - 1 } \sum _ { \kappa \in \tau _ { h } } \left(\eta_{n, \kappa}^{2}+\left\|\mathbf{f}-\mathbf{f}^{k}\right\|_{L^{2}\left(t_{n}, t_{n+1} ; L^{2}(\kappa)^{3}\right)}^{2}\right.\right. \\
& \left.\left.\quad+h_{\kappa}^{2}\left\|\operatorname{div} \mathbf{f}^{k}\right\|_{L^{2}\left(t_{n}, t_{n+1} ; L^{2}(\kappa)^{3}\right)}^{2}+\sum_{e \in \varepsilon_{\kappa}} h_{e}\left\|\left[\left(\mathbf{f}^{k}-\rho_{h} \mathbf{f}^{k}\right) \cdot \mathbf{n}\right]\right\|_{L^{2}\left(t_{n}, t_{n+1} ; L^{2}(e)\right)}^{2}\right)\right\}
\end{aligned}
$$

Proof: For any $q_{h}(t) \in M_{0 h}$, we have

$$
\begin{aligned}
& \left(\nabla\left(p(t)-p_{h}(t)\right), \nabla q(t)\right) \\
& =\left(\mathbf{f}(t)-\mathbf{f}^{k}(t), \nabla q(t)\right)+\left(\mathbf{f}^{k}(t)-\rho_{h} \mathbf{f}^{k}(t), \nabla\left(q(t)-q_{h}(t)\right)\right)+\left(\rho_{h} \mathbf{f}^{k}(t)-\nabla p_{h}(t), \nabla\left(q(t)-q_{h}(t)\right)\right) \\
& =\sum_{\kappa \in \tau_{h}}\left(\int_{\kappa}\left(\mathbf{f}(t)-\mathbf{f}^{k}(t)\right) \nabla q(t)+\int_{\kappa}\left(\mathbf{f}^{k}(t)-\rho_{h} \mathbf{f}^{k}(t)\right) \nabla\left(q(t)-q_{h}(t)\right)+\int_{\kappa}\left(\rho_{h} \mathbf{f}^{k}(t)-\nabla p_{h}(t)\right) \nabla\left(q(t)-q_{h}(t)\right)\right) \\
& =\sum_{\kappa \in \tau_{h}}\left\{\int_{\kappa}\left(\mathbf{f}(t)-\mathbf{f}^{k}(t)\right) \nabla q(t)-\int_{\kappa} \operatorname{div} \mathbf{f}^{k}(t)\left(q(t)-q_{h}(t)\right)\right. \\
& \left.\quad+\frac{1}{2} \sum_{e \in \varepsilon_{\kappa}} \int_{e}\left(\left[\left(\mathbf{f}^{k}(t)-\rho_{h} \mathbf{f}^{k}(t)\right) \cdot \mathbf{n}\right]\left(q(t)-q_{h}(t)\right)+\left[\left(\rho_{h} \mathbf{f}^{k}(t)-\nabla p_{h}(t)\right) \cdot \mathbf{n}\right]\left(q(t)-q_{h}(t)\right)\right)\right\}
\end{aligned}
$$


We take $q(t)=\left(p(t)-p_{h}(t)\right)$ and $q_{h}(t)=R_{h} q(t)$, the image of $q(t)$ by the Clément type regularisation operator, and we obtain:

$$
\begin{aligned}
\|\nabla q(t)\|_{L^{2}(\Omega)}^{2} \leq C \sum_{\kappa \in \tau_{h}}\left\{\frac{1}{2 \varepsilon_{1}}\left(\left\|\mathbf{f}(t)-\mathbf{f}^{k}(t)\right\|_{0, \kappa}^{2}+\frac{1}{2} \varepsilon_{1}\|\nabla q(t)\|_{0, \kappa}^{2}\right)\right. \\
+\left(\frac{1}{2 \varepsilon_{2}} h_{k}^{2}\left\|\operatorname{div} \mathbf{f}^{k}(t)\right\|_{0, \kappa}^{2}+\frac{1}{2} \varepsilon_{2}\|\nabla q(t)\|_{0, \Delta_{\kappa}}^{2}\right) \\
+\sum_{e \in \varepsilon_{\kappa}}\left(\left(\frac{1}{2 \varepsilon_{3}} h_{e}\left\|\left[\left(\mathbf{f}^{k}(t)-\rho_{h} \mathbf{f}^{k}(t)\right) \cdot \mathbf{n}\right]\right\|_{0, e}^{2}+\frac{1}{2} \varepsilon_{3}\|\nabla q(t)\|_{0, \Delta_{e}}^{2}\right)\right. \\
\left.\left.+\left(\frac{1}{2 \varepsilon_{4}} h_{e}\left\|\left[\left(\rho_{h} \mathbf{f}^{k}(t)-\nabla p_{h}(t)\right) \cdot \mathbf{n}\right]\right\|_{0, e}^{2}+\frac{1}{2} \varepsilon_{4}\|\nabla q(t)\|_{0, \Delta_{e}}^{2}\right)\right)\right\}
\end{aligned}
$$

As $\Delta_{e} \subset \Delta_{\kappa}$ and after a suitable choice of $\varepsilon_{i}, i=1,2,3$, w, we integrate between $t_{n}$ and $t_{n+1}$ and we sum over $n$ from 0 to $N-1$ to obtain the result.

Proposition 5.4. The error indicators verify the following optimality conditions

$$
\begin{aligned}
\eta_{n, \kappa}^{2} \leq & C^{\prime}\left(\left|p-p_{h}\right|_{L^{2}\left(t_{n}, t_{n+1} ; H^{1}\left(\Delta_{\kappa}\right)\right)}^{2}+\left\|\mathbf{f}-\mathbf{f}^{k}\right\|_{L^{2}\left(t_{n}, t_{n+1} ; L^{2}\left(\Delta_{\kappa}\right)^{3}\right)}^{2}\right. \\
& \left.+h_{e}^{2}\left\|\operatorname{div} \mathbf{f}^{k}\right\|_{L^{2}\left(t_{n}, t_{n+1} ; L^{2}\left(\Delta_{\kappa}\right)\right)}^{2}+\sum_{e \in \varepsilon_{\kappa}} h_{e}\left\|\left[\left(\mathbf{f}^{k}-\rho_{h} \mathbf{f}^{k}\right) . \mathbf{n}\right]\right\|_{L^{2}\left(t_{n}, t_{n+1} ;\left(L^{2}(e)\right)^{3}\right)}^{2}\right)
\end{aligned}
$$

Proof: We consider the equation (5.5) with $q_{h}=0$ and we take $q(t)=q_{e}(t)=\mathcal{L}_{e}\left(\left[\left(\rho_{h} \mathbf{f}^{k}(t)-\right.\right.\right.$ $\left.\left.\left.\nabla p_{h}(t)\right) \cdot \mathbf{n}\right] \psi_{e}\right)$ :

$$
\begin{aligned}
\int_{\kappa \cup \kappa^{\prime}} \nabla\left(p(t)-p_{h}(t)\right) \nabla q_{e}(t)= & \int_{\kappa \cup \kappa^{\prime}}\left(\mathbf{f}(t)-\mathbf{f}^{k}(t)\right) \nabla q_{e}(t)-\int_{\kappa \cup \kappa^{\prime}} \operatorname{div} \mathbf{f}^{k}(t) q_{e}(t) \\
& +\frac{1}{2} \int_{e}\left(\left[\left(\mathbf{f}^{k}(t)-\rho_{h} \mathbf{f}^{k}(t)\right) \cdot \mathbf{n}\right] q_{e}(t)+\left[\left(\rho_{h} \mathbf{f}^{k}(t)-\nabla p_{h}(t)\right) \cdot \mathbf{n}\right]^{2} \psi_{e}\right)
\end{aligned}
$$

then by using the properties 5.1 and 5.2

$$
\begin{aligned}
\left\|\left[\left(\rho_{h} \mathbf{f}^{k}(t)-\nabla p_{h}(t)\right) \cdot \mathbf{n}\right]\right\|_{0, e}^{2} \leq & C^{\prime}\left(h_{e}^{-1}\left|p(t)-p_{h}(t)\right|_{1, \kappa \cup \kappa^{\prime}}^{2}+h_{e}^{-1}\left\|\mathbf{f}(t)-\mathbf{f}^{k}(t)\right\|_{0, \kappa \cup \kappa^{\prime}}^{2}\right. \\
& \left.+h_{e}\left\|\operatorname{div} \mathbf{f}^{k}(t)\right\|_{0, \kappa \cup \kappa^{\prime}}^{2}+\left\|\left[\left(\mathbf{f}^{k}(t)-\rho_{h} \mathbf{f}^{k}(t)\right) . \mathbf{n}\right]\right\|_{0, e}^{2}\right),
\end{aligned}
$$

multiplying by $h_{e}$, integrating over $t$ from $t_{n}$ to $t_{n+1}$ and summing over $\varepsilon_{\kappa}$, we obtain the result.

Now, let us establish a posteriori error for the velocity. The error function $\mathbf{u}(t)-\mathbf{u}_{h}(t)$ belongs to $H_{0}(\operatorname{curl}, \Omega)$, there exists a function $\lambda(t) \in H_{0}^{1}(\Omega)$ solution of the problem:

$$
\forall \mu \in H_{0}^{1}(\Omega), \quad \int_{\Omega} \nabla \lambda(t) \nabla \mu=\int_{\Omega}\left(\mathbf{u}(t)-\mathbf{u}_{h}(t)\right) \nabla \mu
$$

Then the function $\mathbf{w}(t)=\left(\mathbf{u}(t)-\mathbf{u}_{h}(t)\right)-\nabla \lambda(t)$ belongs to $V_{0}$ and we have curl $\mathbf{w}(t)=\mathbf{c u r l}\left(\mathbf{u}(t)-\mathbf{u}_{h}(t)\right)$. We obtain

$$
\begin{aligned}
& \left\|\mathbf{u}(t)-\mathbf{u}_{h}(t)\right\|_{0, \Omega}^{2}=\|\nabla \lambda(t)\|_{0, \Omega}^{2}+\|\mathbf{w}(t)\|_{0, \Omega}^{2} \\
& \left\|\mathbf{c u r l}\left(\mathbf{u}(t)-\mathbf{u}_{h}(t)\right)\right\|_{0, \Omega}^{2}=\|\mathbf{c u r l} \mathbf{w}(t)\|_{0, \Omega}^{2}
\end{aligned}
$$

In order to find the upper and lower bounds of the velocity, we start by finding the upper and lower bounds of the right hand sides of the last equations. 
For the first term of the right hand side of (5.9), we have for a $t \in\left[t_{n}, t_{n+1}\right]$ and $\forall \mu(t) \in H_{0}^{1}(\Omega)$,

$$
\begin{aligned}
\int_{\Omega} \nabla \lambda(t) \nabla \mu(t) & =\int_{\Omega}(\mathbf{w}(t)+\nabla \lambda(t)) \nabla \mu(t)=\int_{\Omega}\left(\mathbf{u}(t)-\mathbf{u}_{h}(t)\right) \nabla \mu(t) \\
& =-\int_{\Omega} \mathbf{u}_{h}(t) \nabla \mu(t)=-\int_{\Omega} \mathbf{u}_{h}(t) \nabla\left(\mu(t)-\mu_{h}(t)\right) \quad \forall \mu_{h}(t) \in Q_{0 h}
\end{aligned}
$$

then, using the fact that $\operatorname{div} \mathbf{u}_{h}(t)=0$ on every element $\kappa \in \tau_{h}$, we have

$$
\int_{\Omega} \nabla \lambda(t) \nabla \mu(t)=-\frac{1}{2} \sum_{\kappa \in \tau_{h}}\left(\sum_{e \in \varepsilon_{\kappa}} \int_{e}\left[\mathbf{u}_{h}(t) \cdot \mathbf{n}\right]\left(\mu(t)-\mu_{h}(t)\right)\right)
$$

The same steps with $\lambda^{\prime}(t)$ gives:

$$
\int_{\Omega} \nabla \lambda^{\prime}(t) \nabla \mu(t)=-\frac{1}{2} \sum_{\kappa \in \tau_{h}}\left(\sum_{e \in \varepsilon_{\kappa}} \int_{e}\left[\mathbf{u}_{h}^{\prime}(t) \cdot \mathbf{n}\right]\left(\mu(t)-\mu_{h}(t)\right)\right)
$$

with

We introduce the indicators

$$
\mathbf{u}_{h}^{\prime}(t)=\frac{\mathbf{u}_{h}^{n+1}-\mathbf{u}_{h}^{n}}{k} \quad \text { for } t \in\left[t_{n}, t_{n+1}[\text { for all } n=0, \ldots, N-1\right.
$$

$$
\xi_{n, \kappa}^{2}=\sum_{e \in \varepsilon_{\kappa}} h_{e}\left(\left\|\left[\mathbf{u}_{h} \cdot \mathbf{n}\right]\right\|_{L^{\infty}\left(t_{n}, t_{n+1} ; L^{2}(e)\right)}^{2}+\left\|\left[\mathbf{u}_{h}^{\prime} \cdot \mathbf{n}\right]\right\|_{L^{2}\left(t_{n}, t_{n+1} ; L^{2}(e)\right)}^{2}\right)
$$

Theorem 5.5. The following bounds hold

$$
\sum_{n=0}^{N-1}\left(\|\nabla \lambda\|_{L^{\infty}\left(t_{n}, t_{n+1} ; L^{2}(\Omega)^{3}\right)}^{2}+\left\|\nabla \lambda^{\prime}\right\|_{L^{2}\left(t_{n}, t_{n+1} ; L^{2}(\Omega)^{3}\right)}^{2}\right) \leq C_{1}\left(\sum_{n=0}^{N-1} \sum_{\kappa \in \tau_{h}} \xi_{n, \kappa}^{2}\right)
$$

and

$$
\xi_{n, \kappa}^{2} \leq C_{2}\left(\|\nabla \lambda\|_{L^{\infty}\left(t_{n}, t_{n+1} ; L^{2}\left(\Delta_{\kappa}\right)^{3}\right)}^{2}+\left\|\nabla \lambda^{\prime}\right\|_{L^{2}\left(t_{n}, t_{n+1} ; L^{2}\left(\Delta_{\kappa}\right)^{3}\right)}^{2}\right)
$$

Proof: For the upper bound, first we take $\mu=\lambda(t)$ and $\mu_{h}=R_{h} \mu$ in (5.10) and integrate over $t$ from $t_{n}$ to $t_{n+1}$, and second, we take $\mu=\lambda^{\prime}(t)$ and $\mu_{h}=R_{h} \mu$ in (5.11), and we obtain the result after summing over $n$.

In order to find the lower bound, we take in the equation (5.10) (resp. (5.11)), $\mu_{h}=0$ and $\mu=$ $\mathcal{L}_{e}\left(\left[\mathbf{u}_{h}(t) . \mathbf{n}\right] \psi_{e}\right)\left(\right.$ resp. $\left.\mu=\mathcal{L}_{e}\left(\left[\mathbf{u}_{h}^{\prime}(t) . \mathbf{n}\right] \psi_{e}\right)\right)$ and we obtain by using the property 5.2

$$
\left\|\left[\mathbf{u}_{h}(t) . \mathbf{n}\right]\right\|_{0, e}^{2} \leq C_{2}\left(h_{e}^{-1}\|\nabla \lambda(t)\|_{0, \kappa \cup \kappa^{\prime}}^{2}\right) ; \quad\left\|\left[\mathbf{u}_{h}^{\prime}(t) . \mathbf{n}\right]\right\|_{0, e}^{2} \leq C_{2}\left(h_{e}^{-1}\left\|\nabla \lambda^{\prime}(t)\right\|_{0, \kappa \cup \kappa^{\prime}}^{2}\right) ;
$$

which leads, after summing over $e \in \varepsilon_{\kappa}$, to

$$
\xi_{n, \kappa}^{2} \leq C_{2}\left(\|\nabla \lambda\|_{L^{\infty}\left(t_{n}, t_{n+1} ; L^{2}\left(\Delta_{\kappa}\right)^{3}\right)}^{2}+\left\|\nabla \lambda^{\prime}\right\|_{L^{2}\left(t_{n}, t_{n+1} ; L^{2}\left(\Delta_{\kappa}\right)^{3}\right)}^{2}\right)
$$

Now, we will bound the second and third terms of the right hand side of (5.9):

For $t \in\left[t_{n}, t_{n+1}\left[, \forall \mathbf{v}(t) \in H_{0}(\operatorname{curl}, \Omega)\right.\right.$ :

$$
\begin{aligned}
\left(\frac{d}{d t}(\mathbf{u}\right. & \left.\left.-\mathbf{u}_{h}\right)(t), \mathbf{v}(t)\right)+\nu\left(\operatorname{curl}\left(\mathbf{u}(t)-\mathbf{u}_{h}(t)\right), \operatorname{curl} \mathbf{v}(t)\right)+\left(\nabla\left(p(t)-p_{h}(t)\right), \mathbf{v}(t)\right) \\
& =(\mathbf{f}(t), \mathbf{v}(t))-\frac{1}{k}\left(\mathbf{u}_{h}^{n+1}-\mathbf{u}_{h}^{n}, \mathbf{v}(t)\right)-\nu\left(\operatorname{curl} \mathbf{u}_{h}(t), \operatorname{curl} \mathbf{v}(t)\right)-\left(\nabla p_{h}(t), \mathbf{v}(t)\right)
\end{aligned}
$$

We introduce the residual $R\left(\mathbf{u}_{h}\right) \in L^{2}\left(0, T ;\left(H_{0}(\mathbf{c u r l}, \Omega)\right)^{\prime}\right)$ : for $t \in\left[t_{n}, t_{n+1}\left[, \forall \mathbf{v}(t) \in H_{0}(\mathbf{c u r l}, \Omega)\right.\right.$

$$
\left\langle R\left(\mathbf{u}_{h}\right)(t), \mathbf{v}(t)\right\rangle=(\mathbf{f}(t), \mathbf{v}(t))-\left(\frac{d \mathbf{u}_{h}}{d t}(t), \mathbf{v}(t)\right)-\nu\left(\operatorname{curl} \mathbf{u}_{h}(t), \operatorname{curl} \mathbf{v}(t)\right)-\left(\nabla p_{h}(t), \mathbf{v}(t)\right)
$$

Using (3.6), we introduce the space residual $R^{h}$ and the time residual $R^{\tau}$ :

$$
R\left(\mathbf{u}_{h}\right)=\mathbf{f}-\mathbf{f}\left(t_{n+1}\right)+R^{h}\left(\mathbf{u}_{h}\right)+R^{\tau}\left(\mathbf{u}_{h}\right)
$$


with, for $t \in\left[t_{n}, t_{n+1}\left[, \forall \mathbf{v}_{h}(t) \in H_{0}(\operatorname{curl}, \Omega)\right.\right.$ :

$$
\begin{aligned}
\left\langle R^{h}\left(\mathbf{u}_{h}\right)(t), \mathbf{v}(t)\right\rangle= & \left(\mathbf{f}\left(t_{n+1}\right)-\frac{1}{k}\left(\mathbf{u}_{h}^{n+1}-\mathbf{u}_{h}^{n}\right)-\nabla p_{h}^{n+1}, \mathbf{v}(t)\right)-\nu\left(\mathbf{c u r l} \mathbf{u}_{h}^{n+1}, \mathbf{c u r l} \mathbf{v}(t)\right) \\
= & \left(\mathbf{f}\left(t_{n+1}\right)-\frac{1}{k}\left(\mathbf{u}_{h}^{n+1}-\mathbf{u}_{h}^{n}\right)-\nabla p_{h}^{n+1}, \mathbf{v}(t)-\mathbf{v}_{h}(t)\right)-\nu\left(\mathbf{c u r l} \mathbf{u}_{h}^{n+1}, \mathbf{c u r l}\left(\mathbf{v}(t)-\mathbf{v}_{h}(t)\right)\right. \\
= & \sum_{\kappa \in \tau_{h}}\left\{\int_{\kappa}\left(\mathbf{f}\left(t_{n+1}\right)-\frac{1}{k}\left(\mathbf{u}_{h}^{n+1}-\mathbf{u}_{h}^{n}\right)-\nabla p_{h}^{n+1}\right)\left(\mathbf{v}(t)-\mathbf{v}_{h}(t)\right)\right. \\
+ & \left.\frac{\nu}{2} \sum_{e \in \varepsilon_{\kappa}} \int_{e}\left(\left[\mathbf{c u r l} \mathbf{u}_{h}^{n+1} \times \mathbf{n}\right]\right)\left(\mathbf{v}(t)-\mathbf{v}_{h}(t)\right)\right\} \\
\left\langle R^{\tau}\left(\mathbf{u}_{h}\right)(t), \mathbf{v}(t)\right\rangle= & \nu\left(\mathbf{c u r l}\left(\mathbf{u}_{h}^{n+1}-\mathbf{u}_{h}(t)\right), \mathbf{c u r l} \mathbf{v}(t)\right)+\left(\nabla\left(p_{h}^{n+1}-p_{h}(t)\right), \mathbf{v}(t)\right) \\
= & \sum_{\kappa \in \tau_{h}}\left\{\int_{\kappa} \nabla\left(p_{h}^{n+1}-p_{h}(t)\right) \mathbf{v}(t)+\nu \int_{\kappa} \mathbf{c u r l}\left(\mathbf{u}_{h}^{n+1}-\mathbf{u}_{h}(t)\right) \mathbf{c u r l} \mathbf{v}(t)\right\}
\end{aligned}
$$

We introduce, for each $\kappa \in \tau_{h}$, the indicators:

$$
\begin{aligned}
& \gamma_{n, \kappa}^{2}=h_{\kappa}^{2}\left\|\rho_{h} \mathbf{f}\left(t_{n+1}\right)-\frac{1}{k}\left(\mathbf{u}_{h}^{n+1}-\mathbf{u}_{h}^{n}\right)-\nabla p_{h}^{n+1}\right\|_{0, \kappa}^{2}+\sum_{e \in \varepsilon_{\kappa}} h_{e}\left\|\left[\operatorname{curl} \mathbf{u}_{h}^{n+1} \times \mathbf{n}\right]\right\|_{0, e}^{2} \\
& \delta_{n, \kappa}^{2}=\left\|\operatorname{curl}\left(\mathbf{u}_{h}^{n+1}-\mathbf{u}_{h}^{n}\right)\right\|_{0, \kappa}^{2}
\end{aligned}
$$

Theorem 5.6. We have the upper bound

$$
\begin{aligned}
& \|\mathbf{w}\|_{L^{\infty}\left(0, t_{m} ; L^{2}(\Omega)^{3}\right)}^{2}+\|\mathbf{c u r l} \mathbf{w}\|_{L^{2}\left(0, t_{m} ; L^{2}(\Omega)^{3}\right)}^{2}+\left\|\mathbf{w}^{\prime}\right\|_{L^{2}\left(0, t_{m} ; L^{2}(\Omega)^{3}\right)}^{2} \\
& \leq C \sum_{n=0}^{m-1}\left(\left\|\mathbf{f}-\mathbf{f}\left(t_{n+1}\right)\right\|_{L^{2}\left(t_{n}, t_{n+1} ; L^{2}(\Omega)^{3}\right)}^{2}+\sum_{\kappa \in \tau_{h}}\left(k h_{\kappa}^{2}\left\|\rho_{h} \mathbf{f}\left(t_{n+1}\right)-\mathbf{f}\left(t_{n+1}\right)\right\|_{L^{2}(\kappa)^{3}}^{2}+k \gamma_{n, \kappa}^{2}+k \delta_{n, \kappa}^{2}\right)\right)
\end{aligned}
$$

Proof: First, we consider the equations (5.15) and (5.17) and the definition of $R\left(\mathbf{u}_{h}\right)$, we replace $\mathbf{u}(t)-\mathbf{u}_{h}(t)=\mathbf{w}(t)+\nabla \lambda(t)$ and $\mathbf{v}(t)=\mathbf{w}(t)$, integrate over $t$ from $t_{n}$ to $t_{n+1}$, sum over $n$ from 0 to $m-1$ and using the theorem 2.2 :

$$
\begin{gathered}
\frac{1}{2}\left\|\mathbf{w}\left(t_{m}\right)\right\|_{0, \Omega}^{2}+\nu\|\mathbf{c u r l} \mathbf{w}\|_{L^{2}\left(0, t_{m} ; L^{2}(\Omega)^{3}\right)}^{2} \leq C\left(\sum_{n=0}^{m-1}\left\|\mathbf{f}-\mathbf{f}\left(t_{n+1}\right)\right\|_{L^{2}\left(t_{n}, t_{n+1} ; L^{2}(\Omega)^{3}\right)}\right. \\
\left.+\left\|R^{h}\left(\mathbf{u}_{h}\right)\right\|_{L^{2}\left(0, t_{m} ; V_{0}^{\prime}\right)}+\left\|R^{\tau}\left(\mathbf{u}_{h}\right)\right\|_{L^{2}\left(0, t_{m} ; V_{0}^{\prime}\right)}\right)\|\mathbf{c u r l} \mathbf{w}\|_{L^{2}\left(0, t_{m} ; L^{2}(\Omega)^{3}\right)}
\end{gathered}
$$

which leads to

$$
\begin{aligned}
& \|\mathbf{w}\|_{L^{\infty}\left(0, T ; L^{2}(\Omega)^{3}\right)}^{2}+\|\mathbf{c u r l} \mathbf{w}\|_{L^{2}\left(0, T ; L^{2}(\Omega)^{3}\right)}^{2} \\
& \leq C^{\prime}\left(\sum_{n=0}^{N-1}\left\|\mathbf{f}-\mathbf{f}\left(t_{n+1}\right)\right\|_{L^{2}\left(t_{n}, t_{n+1} ; L^{2}(\Omega)^{3}\right)}^{2}+\left\|R^{h}\left(\mathbf{u}_{h}\right)\right\|_{L^{2}\left(0, T ; V_{0}^{\prime}\right)}^{2}+\left\|R^{\tau}\left(\mathbf{u}_{h}\right)\right\|_{L^{2}\left(0, T ; V_{0}^{\prime}\right)}^{2}\right)
\end{aligned}
$$

We repeat the same procedure with $\mathbf{v}(t)=\mathbf{w}^{\prime}(t)$ and combining the tow steps:

$$
\begin{aligned}
& \|\mathbf{w}\|_{L^{\infty}\left(0, T ; L^{2}(\Omega)^{3}\right)}^{2}+\|\mathbf{c u r l} \mathbf{w}\|_{L^{2}\left(0, T ; L^{2}(\Omega)^{3}\right)}^{2}+\left\|\mathbf{w}^{\prime}\right\|_{L^{2}\left(0, T ; L^{2}(\Omega)^{3}\right)}^{2} \\
& \leq C^{\prime \prime}\left(\sum_{n=0}^{N-1}\left\|\mathbf{f}-\mathbf{f}\left(t_{n+1}\right)\right\|_{L^{2}\left(t_{n}, t_{n+1} ; L^{2}(\Omega)^{3}\right)}^{2}+\left\|R^{h}\left(\mathbf{u}_{h}\right)\right\|_{L^{2}\left(0, T ; V_{0}^{\prime}\right)}^{2}+\left\|R^{\tau}\left(\mathbf{u}_{h}\right)\right\|_{L^{2}\left(0, T ; V_{0}^{\prime}\right)}^{2}\right)
\end{aligned}
$$

To find the upper bound of $\mathbf{w}$, it suffices to find the upper bound of the operator $R^{h}$ and $R^{\tau}$.

We take in the equation (5.18) $\mathbf{v}(t) \in V_{0}$ and $\mathbf{v}_{h}(t)=\mathcal{R}_{h} \mathbf{v}(t)$, we integrate over $t$ from $t_{n}$ to $t_{n+1}$ and we sum over $n$ from 0 to $N-1$. We obtain, using the properties of $\mathcal{R}_{h}$, the upper following bound:

$$
\left\|R^{h}\left(\mathbf{u}_{h}\right)\right\|_{L^{2}\left(0, T ; V_{0}^{\prime}\right)}^{2} \leq C_{1} \sum_{n=0}^{N-1} \sum_{\kappa \in \tau_{h}}\left(k h_{\kappa}^{2}\left\|\rho_{h} \mathbf{f}\left(t_{n+1}\right)-\mathbf{f}\left(t_{n+1}\right)\right\|_{L^{2}(\kappa)^{3}}^{2}+k \gamma_{n, \kappa}^{2}\right)
$$


Similarly, we take the equation (5.19) with $\mathbf{v}(t) \in V_{0}$; we integrate over $t$ from $t_{n}$ to $t_{n+1}$ and we sum over $n$ from 0 to $N-1$. We obtain:

$$
\begin{aligned}
\left\|R^{\tau}\left(\mathbf{u}_{h}\right)\right\|_{L^{2}\left(0, T ; V_{0}^{\prime}\right)}^{2} & \leq C_{2} \sum_{n=0}^{N-1} \sum_{\kappa \in \tau_{h}} \int_{t_{n}}^{t_{n+1}} \frac{\left(t_{n+1}-t\right)^{2}}{k^{2}}\left\|\operatorname{curl}\left(\mathbf{u}_{h}^{n+1}-\mathbf{u}_{h}^{n}\right)\right\|_{L^{2}(\kappa)^{3}}^{2} \\
& \leq \frac{C_{2}}{3} \sum_{n=0}^{N-1} \sum_{\kappa \in \tau_{h}} k \delta_{n, \kappa}^{2}
\end{aligned}
$$

The formulas (5.23), (5.24) and (5.25) give the result.

Next, we will show some properties of the operator $R^{h}$. To do that, we introduce, for any element $\kappa$, the operators:

$$
\begin{aligned}
R_{n, \kappa}^{h} & =\rho_{h} \mathbf{f}\left(t_{n+1}\right)-\frac{1}{k}\left(\mathbf{u}_{h}^{n+1}-\mathbf{u}_{h}^{n}\right)-\nabla p_{h}^{n+1} \\
R_{n, e}^{h} & =\left[\mathbf{c u r l} \mathbf{u}_{h}^{n+1} \times \mathbf{n}\right]_{e}
\end{aligned}
$$

and, for some $\alpha_{1}>$ and $\alpha_{2}>0$ :

$$
\theta_{n}=\sum_{\kappa \in \tau_{h}} \theta_{n, \kappa} \quad \text { where } \quad \theta_{n, \kappa}=\alpha_{1} h_{\kappa}^{2} R_{n, \kappa}^{h} \psi_{\kappa}+\alpha_{2} \sum_{e \in \varepsilon_{\kappa}} h_{e} \mathcal{L}_{e}\left(R_{n, e}^{h} \psi_{e}\right)
$$

Theorem 5.7. There exist constants $C_{1 \dagger}$ and $C_{2 \dagger}$ such that, on each interval $\left[t_{n}, t_{n+1}\right]$, the following estimates, for some $\theta_{n}$, hold:

$$
\begin{gathered}
\left\|\theta_{n}\right\|_{0, \Omega}^{2} \leq C_{1 \dagger} \sum_{\kappa \in \tau_{h}} h_{\kappa}^{2} \gamma_{n, \kappa}^{2} \\
\left\|\mathbf{c u r l} \theta_{n}\right\|_{0, \Omega}^{2} \leq C_{2 \dagger} \sum_{\kappa \in \tau_{h}} \gamma_{n, \kappa}^{2} \\
\left\langle R^{h}\left(\mathbf{u}_{h}\right), \theta_{n}\right\rangle \geq\left(\mathbf{f}\left(t_{n+1}\right)-\rho_{h} \mathbf{f}\left(t_{n+1}\right), \theta_{n}\right)+\sum_{\kappa \in \tau_{h}} \gamma_{n, \kappa}^{2}
\end{gathered}
$$

Proof: Using the properties 5.2 and 5.1 and the fact that $h_{e} \leq h_{\kappa}$, we have:

$$
\begin{aligned}
\left\|\theta_{n}\right\|_{0, \Omega}^{2}= & \sum_{\kappa \in \tau_{h}}\left\|\theta_{n}\right\|_{0, \kappa}^{2} \\
= & \sum_{\kappa \in \tau_{h}}\left(\alpha_{1}^{2} h_{\kappa}^{4}\left\|\left(R_{n, \kappa}^{h} \psi_{\kappa}\right)\right\|_{0, \kappa}^{2}+\alpha_{2}^{2} \sum_{e \in \varepsilon_{\kappa}} \sum_{e^{\prime} \in \varepsilon_{\kappa}} h_{e} h_{e^{\prime}}\left(\left(\mathcal{L}\left(R_{n, e}^{h} \psi_{e}\right)\right),\left(\mathcal{L}\left(R_{n, e^{\prime}}^{h} \psi_{e^{\prime}}\right)\right)\right)_{\kappa}\right. \\
& \left.+2 \alpha_{1} \alpha_{2} \sum_{e \in \varepsilon_{\kappa}} h_{\kappa}^{2} h_{e}\left(\left(R_{n, \kappa}^{h} \psi_{\kappa}\right),\left(\mathcal{L}\left(R_{n, e}^{h} \psi_{e}\right)\right)\right)_{\kappa}\right) \\
\leq & C_{1 \dagger} \sum_{\kappa \in \tau_{h}} h_{\kappa}^{2} \gamma_{n, \kappa}^{2}
\end{aligned}
$$

and

$$
\begin{aligned}
\left\|\operatorname{curl} \theta_{n}\right\|_{0, \Omega}^{2}= & \sum_{\kappa \in \tau_{h}}\left\|\operatorname{curl} \theta_{n}\right\|_{0, \kappa}^{2} \\
= & \sum_{\kappa \in \tau_{h}}\left(\alpha_{1}^{2} h_{\kappa}^{4}\left\|\operatorname{curl}\left(R_{n, \kappa}^{h} \psi_{\kappa}\right)\right\|_{0, \kappa}^{2}+\alpha_{2}^{2} \sum_{e \in \varepsilon_{\kappa}} \sum_{e^{\prime} \in \varepsilon_{\kappa}} h_{e} h_{e^{\prime}}\left(\operatorname{curl}\left(\mathcal{L}\left(R_{n, e}^{h} \psi_{e}\right)\right), \operatorname{curl}\left(\mathcal{L}\left(R_{n, e^{\prime}}^{h} \psi_{e^{\prime}}\right)\right)\right)_{\kappa}\right. \\
& \left.+2 \alpha_{1} \alpha_{2} \sum_{e \in \varepsilon_{\kappa}} h_{\kappa}^{2} h_{e}\left(\operatorname{curl}\left(R_{n, \kappa}^{h} \psi_{\kappa}\right), \operatorname{curl}\left(\mathcal{L}\left(R_{n, e}^{h} \psi_{e}\right)\right)\right)_{\kappa}\right) \\
\leq & C_{2 \dagger} \sum_{\kappa \in \tau_{h}} \gamma_{n, \kappa}^{2}
\end{aligned}
$$


Furthermore, we consider the equation (5.18) with $\mathbf{v}(t)=\theta_{n}$ and $\mathbf{v}_{h}(t)=0$ :

$$
\begin{aligned}
\left\langle R^{h}\left(\mathbf{u}_{h}\right)(t), \theta_{n}\right\rangle= & \left(\mathbf{f}\left(t_{n+1}\right)-\rho_{h} \mathbf{f}\left(t_{n+1}\right), \theta_{n}\right)+\sum_{\kappa \in \tau_{h}}\left(R_{n, \kappa}^{h}, \theta_{n}\right)_{\kappa}+\frac{\nu}{2} \sum_{\kappa \in \tau_{h}} \sum_{e \in \varepsilon_{\kappa}}\left(R_{n, e}^{h}, \theta_{n}\right)_{e} \\
= & \left(\mathbf{f}\left(t_{n+1}\right)-\rho_{h} \mathbf{f}\left(t_{n+1}\right), \theta_{n}\right)+\alpha_{1} \sum_{\kappa \in \tau_{h}} h_{\kappa}^{2}\left(R_{n, \kappa}^{h}, R_{n, \kappa}^{h} \psi_{\kappa}\right)_{\kappa} \\
& +2 \alpha_{2} \sum_{\kappa \in \tau_{h}} \sum_{e \in \varepsilon_{\kappa}} h_{e}\left(R_{n, \kappa}^{h}, \mathcal{L}_{e}\left(R_{n, e}^{h} \psi_{e}\right)\right)_{\kappa}+\nu \alpha_{2} \sum_{\kappa \in \tau_{h}} \sum_{e \in \varepsilon_{\kappa}} h_{e}\left(R_{n, e}^{h}, R_{n, e}^{h} \psi_{e}\right)_{e}
\end{aligned}
$$

We have by using the properties 5.2 and 5.1, and remarking that $h_{e} \leq h_{\kappa}$ :

$$
\begin{aligned}
\left\langle R^{h}\left(\mathbf{u}_{h}\right)(t), \theta_{n}\right\rangle \geq & \left(\mathbf{f}\left(t_{n+1}\right)-\rho_{h} \mathbf{f}\left(t_{n+1}\right), \theta_{n}\right)+C_{1} \alpha_{1} \sum_{\kappa \in \tau_{h}} h_{\kappa}^{2}\left\|R_{n, \kappa}^{h}\right\|_{0, \kappa}^{2} \\
& -2 \alpha_{2} C_{2} \sum_{\kappa \in \tau_{h}} \sum_{e \in \varepsilon_{\kappa}} h_{\kappa} h_{e}^{1 / 2}\left\|R_{n, \kappa}^{h}\right\|_{0, \kappa}\left\|R_{n, e}^{h}\right\|_{0, e}+\nu \alpha_{2} C_{3} \sum_{\kappa \in \tau_{h}} \sum_{e \in \varepsilon_{\kappa}} h_{e}\left\|R_{n, e}^{h}\right\|_{0, e}^{2} \\
\geq & \left(\mathbf{f}\left(t_{n+1}\right)-\rho_{h} \mathbf{f}\left(t_{n+1}\right), \theta_{n}\right)+C_{1} \alpha_{1} \sum_{\kappa \in \tau_{h}} h_{\kappa}^{2}\left\|R_{n, \kappa}^{h}\right\|_{0, \kappa}^{2}+\nu \alpha_{2} C_{3} \sum_{\kappa \in \tau_{h}} \sum_{e \in \varepsilon_{\kappa}} h_{e}\left\|R_{n, e}^{h}\right\|_{0, e}^{2} \\
& -2 \alpha_{2} C_{2} \sum_{\kappa \in \tau_{h}} \sum_{e \in \varepsilon_{\kappa}}\left\{\frac{1}{2 \varepsilon} h_{\kappa}^{2}\left\|R_{n, \kappa}^{h}\right\|_{0, \kappa}^{2}+\frac{1}{2} \varepsilon h_{e}\left\|R_{n, e}^{h}\right\|_{0, e}^{2}\right\} \\
\geq & \left(\mathbf{f}\left(t_{n+1}\right)-\rho_{h} \mathbf{f}\left(t_{n+1}\right), \theta_{n}\right)+\left(C_{1} \alpha_{1}-\frac{4 \alpha_{2} C_{2}}{\varepsilon}\right) \sum_{\kappa \in \tau_{h}} h_{\kappa}^{2}\left\|R_{n, \kappa}^{h}\right\|_{0, \kappa}^{2} \\
& +\alpha_{2}\left(\nu C_{3}-C_{2} \varepsilon\right) \sum_{\kappa \in \tau_{h}} \sum_{e \in \varepsilon_{\kappa}} h_{e}\left\|R_{n, e}^{h}\right\|_{0, e}^{2}
\end{aligned}
$$

We choice $\varepsilon=\frac{\nu C_{3}}{2 C_{2}}, \alpha_{2}=\frac{2}{\nu C_{3}}$ and $\alpha_{1}=\frac{1}{C_{1}}\left(1+\frac{16 C_{2}^{2}}{\nu C_{3}^{2}}\right)$ to obtain the third estimate.

Theorem 5.8. The following upper bound estimate holds for a positive constant $c_{1}$

$$
\begin{aligned}
\sum_{\kappa \in \tau_{h}} k \delta_{n, \kappa}^{2} \leq & c_{1}\left\{\left\|\mathbf{u}^{\prime}-\mathbf{u}_{h}^{\prime}\right\|_{L^{2}\left(t_{n}, t_{n+1} ; L^{2}(\Omega)^{3}\right)}^{2}+\left\|\nabla\left(p-p_{h}\right)\right\|_{L^{2}\left(t_{n}, t_{n+1} ; L^{2}(\Omega)^{3}\right)}^{2}\right. \\
& \left.+\left\|\mathbf{c u r l}\left(\mathbf{u}-\mathbf{u}_{h}\right)\right\|_{L^{2}\left(t_{n}, t_{n+1} ; L^{2}(\Omega)^{3}\right)}^{2}+\left\|\mathbf{f}-\mathbf{f}\left(t_{n+1}\right)\right\|_{L^{2}\left(t_{n}, t_{n+1} ; L^{2}(\Omega)^{3}\right)}^{2}\right\}
\end{aligned}
$$

Proof: We take the definition of $R^{\tau}$ with $\mathbf{v}(t)=\mathbf{v}_{h}=\left(\mathbf{u}_{h}^{n+1}-\mathbf{u}_{h}^{n}\right) \in V_{0 h}$, we integrate from $t_{n}$ to $t_{n+1}$ and we obtain:

$$
\begin{aligned}
\nu \frac{k}{2}\left\|\operatorname{curl}\left(\mathbf{u}_{h}^{n+1}-\mathbf{u}_{h}^{n}\right)\right\|_{0, \Omega}^{2}= & \int_{t_{n}}^{t_{n+1}}\left\langle R^{\tau}\left(\mathbf{u}_{h}\right)(t), \mathbf{v}_{h}\right\rangle-\int_{t_{n}}^{t_{n+1}}\left(\nabla\left(p_{h}^{n+1}-p_{h}(t)\right), \mathbf{v}_{h}\right) \\
\leq & \int_{t_{n}}^{t_{n+1}}\left|\left\langle R\left(\mathbf{u}_{h}\right)(t), \mathbf{v}_{h}\right\rangle\right|+\int_{t_{n}}^{t_{n+1}}\left|\left\langle R^{h}\left(\mathbf{u}_{h}\right)(t), \mathbf{v}_{h}\right\rangle\right| \\
& +\int_{t_{n}}^{t_{n+1}}\left|\left(\mathbf{f}(t)-\mathbf{f}\left(t_{n+1}\right), \mathbf{v}_{h}\right)\right|+\int_{t_{n}}^{t_{n+1}}\left|\left(\nabla\left(p_{h}^{n+1}-p_{h}(t)\right), \mathbf{v}_{h}\right)\right|
\end{aligned}
$$

We will bound terms of the right side. We remark that $\left(\nabla\left(p_{h}^{n+1}-p_{h}(t)\right), \mathbf{v}_{h}\right)=0$. The definition of $R\left(\mathbf{u}_{h}\right)$ and $R^{h}\left(\mathbf{u}_{h}\right)$, and the fact that $\mathbf{v}_{h} \in V_{0 h}$ give

$$
\left\langle R^{h}\left(\mathbf{u}_{h}\right)(t), \mathbf{v}_{h}\right\rangle=0
$$


and

$$
\begin{aligned}
\int_{t_{n}}^{t_{n+1}}\left|\left\langle R\left(\mathbf{u}_{h}\right)(t), \mathbf{v}_{h}\right\rangle\right| & \leq\left(C\left\|\mathbf{u}^{\prime}-\mathbf{u}_{h}^{\prime}\right\|_{L^{2}\left(t_{n}, t_{n+1} ; L^{2}(\Omega)^{3}\right)}+C\left\|\nabla\left(p-p_{h}\right)\right\|_{L^{2}\left(t_{n}, t_{n+1} ; L^{2}(\Omega)^{3}\right)}\right. \\
& \left.+\nu\left\|\operatorname{curl}\left(\mathbf{u}-\mathbf{u}_{h}\right)\right\|_{L^{2}\left(t_{n}, t_{n+1} ; L^{2}(\Omega)^{3}\right)}\right)\left\|\operatorname{curl} \mathbf{v}_{h}\right\|_{L^{2}\left(t_{n}, t_{n+1} ; L^{2}(\Omega)^{3}\right)}
\end{aligned}
$$

and

$$
\begin{aligned}
\left.\int_{t_{n}}^{t_{n+1}} \mid \mathbf{f}(t)-\mathbf{f}\left(t_{n+1}\right), \mathbf{v}_{h}\right) \mid & \leq \|\left(\mathbf{f}-\mathbf{f}\left(t_{n+1}\right)\left\|_{L^{2}\left(t_{n}, t_{n+1} ; L^{2}(\Omega)^{3}\right)}\right\| \mathbf{v}_{h} \|_{L^{2}\left(t_{n}, t_{n+1} ; L^{2}(\Omega)^{3}\right)}\right. \\
& \leq C \|\left(\mathbf{f}-\mathbf{f}\left(t_{n+1}\right)\left\|_{L^{2}\left(t_{n}, t_{n+1} ; L^{2}(\Omega)^{3}\right)}\right\| \mathbf{c u r l} \mathbf{v}_{h} \|_{L^{2}\left(t_{n}, t_{n+1} ; L^{2}(\Omega)^{3}\right)}\right.
\end{aligned}
$$

We deduce:

$$
\begin{aligned}
\frac{\nu}{\sqrt{2}} k^{1 / 2}\left\|\operatorname{curl}\left(\mathbf{u}_{h}^{n+1}-\mathbf{u}_{h}^{n}\right)\right\|_{0, \Omega} \leq & C^{\prime}\left\{\left\|\mathbf{u}^{\prime}-\mathbf{u}_{h}^{\prime}\right\|_{L^{2}\left(t_{n}, t_{n+1} ; L^{2}(\Omega)^{3}\right)}+\left\|\nabla\left(p-p_{h}\right)\right\|_{L^{2}\left(t_{n}, t_{n+1} ; L^{2}(\Omega)^{3}\right)}\right. \\
& \left.+\nu\left\|\mathbf{c u r l}\left(\mathbf{u}-\mathbf{u}_{h}\right)\right\|_{L^{2}\left(t_{n}, t_{n+1} ; L^{2}(\Omega)^{3}\right)}+\left\|\mathbf{f}-\mathbf{f}\left(t_{n+1}\right)\right\|_{L^{2}\left(t_{n}, t_{n+1} ; L^{2}(\Omega)^{3}\right)}\right\}
\end{aligned}
$$

which leads to the result.

Theorem 5.9. The following upper bound estimate holds

$$
\begin{aligned}
\sum_{\kappa \in \tau_{h}} k \gamma_{n, \kappa}^{2} & \leq c_{2}\left\{\left\|\mathbf{f}\left(t_{n+1}\right)-\rho_{h} \mathbf{f}\left(t_{n+1}\right)\right\|_{L^{2}\left(t_{n}, t_{n+1} ; L^{2}(\Omega)^{3}\right)}^{2}+\left\|\mathbf{f}-\mathbf{f}\left(t_{n+1}\right)\right\|_{L^{2}\left(t_{n}, t_{n+1} ; L^{2}(\Omega)^{3}\right)}^{2}\right. \\
& +\left\|\mathbf{f}\left(t_{n+1}\right)-\mathbf{f}\left(t_{n}\right)\right\|_{L^{2}\left(t_{n}, t_{n+1} ; L^{2}(\Omega)^{3}\right)}^{2}+\left\|\mathbf{u}^{\prime}-\mathbf{u}_{h}^{\prime}\right\|_{L^{2}\left(t_{n}, t_{n+1} ; L^{2}(\Omega)^{3}\right)}^{2}+\left\|\nabla\left(p-p_{h}\right)\right\|_{L^{2}\left(t_{n}, t_{n+1} ; L^{2}(\Omega)^{3}\right)}^{2} \\
& \left.+\left\|\mathbf{c u r l}\left(\mathbf{u}-\mathbf{u}_{h}\right)\right\|_{L^{2}\left(t_{n}, t_{n+1} ; L^{2}(\Omega)^{3}\right)}^{2}\right\}
\end{aligned}
$$

Proof: We consider the inequality (5.29):

$$
\begin{aligned}
\sum_{\kappa \in \tau_{h}} \gamma_{n, \kappa}^{2} & \leq\left\langle R^{h}\left(\mathbf{u}_{h}\right), \theta_{n}\right\rangle-\left(\mathbf{f}\left(t_{n+1}\right)-\rho_{h} \mathbf{f}\left(t_{n+1}\right), \theta_{n}\right) \\
& \leq\left\langle R\left(\mathbf{u}_{h}\right), \theta_{n}\right\rangle-\left\langle R^{\tau}\left(\mathbf{u}_{h}\right), \theta_{n}\right\rangle-\left(\mathbf{f}(t)-\mathbf{f}\left(t_{n+1}\right), \theta_{n}\right)-\left(\mathbf{f}\left(t_{n+1}\right)-\rho_{h} \mathbf{f}\left(t_{n+1}\right), \theta_{n}\right)
\end{aligned}
$$

We integrate the resulting inequality from $t_{n}$ to $t_{n+1}$. We thus arrive at:

$$
\begin{aligned}
\sum_{\kappa \in \tau_{h}} k \gamma_{n, \kappa}^{2} \leq & \int_{t_{n}}^{t_{n+1}}\left\langle R\left(\mathbf{u}_{h}\right), \theta_{n}\right\rangle-\int_{t_{n}}^{t_{n+1}}\left\langle R^{\tau}\left(\mathbf{u}_{h}\right), \theta_{n}\right\rangle-\int_{t_{n}}^{t_{n+1}}\left(\mathbf{f}(t)-\mathbf{f}\left(t_{n+1}\right), \theta_{n}\right) \\
& -\int_{t_{n}}^{t_{n+1}}\left(\mathbf{f}\left(t_{n+1}\right)-\rho_{h} \mathbf{f}\left(t_{n+1}\right), \theta_{n}\right)
\end{aligned}
$$

Since $\theta_{n}$ is constant with respect to time, we obtain by using theorem 5.7 and remarking that $\| \nabla\left(p_{h}^{n+1}-\right.$ $\left.p_{h}^{n}\right)\left\|_{0, \Omega} \leq\right\| f\left(t_{n+1}\right)-f\left(t_{n}\right) \|_{0, \Omega}:$

$$
\begin{aligned}
\int_{t_{n}}^{t_{n+1}}\left\langle R^{\tau}\left(\mathbf{u}_{h}\right), \theta_{n}\right\rangle= & \nu\left(\operatorname{curl}\left(\mathbf{u}_{h}^{n+1}-\mathbf{u}_{h}^{n}\right), \operatorname{curl} \theta_{n}\right) \int_{t_{n}}^{t_{n+1}}\left(\frac{t_{n+1}-t}{k}\right) d t \\
& +\left(\nabla\left(p_{h}^{n+1}-p_{h}^{n}\right), \theta_{n}\right) \int_{t_{n}}^{t_{n+1}}\left(\frac{t_{n+1}-t}{k}\right) d t \\
\leq & \frac{1}{2} \nu \sqrt{C_{2 \dagger}} k^{1 / 2}\left\|\operatorname{curl}\left(\mathbf{u}_{h}^{n+1}-\mathbf{u}_{h}^{n}\right)\right\|_{0, \Omega}\left(\sum_{\kappa \in \tau_{h}} k \gamma_{n, \kappa}^{2}\right)^{1 / 2} \\
& +\frac{1}{2} \sqrt{C_{1 \dagger}} k^{1 / 2}\left\|\mathbf{f}\left(t_{n+1}\right)-\mathbf{f}\left(t_{n}\right)\right\|_{0, \Omega}\left(\sum_{\kappa \in \tau_{h}} k h_{\kappa}^{2} \gamma_{n, \kappa}^{2}\right)^{1 / 2}
\end{aligned}
$$


and

$$
\begin{aligned}
\int_{t_{n}}^{t_{n+1}}\left\langle R\left(\mathbf{u}_{h}\right), \theta_{n}\right\rangle & \leq\left(\left\|\mathbf{u}^{\prime}-\mathbf{u}_{h}^{\prime}\right\|_{L^{2}\left(t_{n}, t_{n+1} ; L^{2}(\Omega)^{3}\right)}+\left\|\nabla\left(p-p_{h}\right)\right\|_{L^{2}\left(t_{n}, t_{n+1} ; L^{2}(\Omega)^{3}\right)}\right) \sqrt{C_{1 \dagger}}\left(\sum_{\kappa \in \tau_{h}} k h_{k}^{2} \gamma_{n, \kappa}^{2}\right)^{1 / 2} \\
& +\nu \sqrt{C_{2 \dagger}}\left\|\operatorname{curl}\left(\mathbf{u}-\mathbf{u}_{h}\right)\right\|_{L^{2}\left(t_{n}, t_{n+1} ; L^{2}(\Omega)^{3}\right)}\left(\sum_{\kappa \in \tau_{h}} k \gamma_{n, \kappa}^{2}\right)^{1 / 2}
\end{aligned}
$$

Combining the last estimates and using the previous theorem give:

$$
\begin{aligned}
\sum_{\kappa \in \tau_{h}} k \gamma_{n, \kappa}^{2} & \leq \hat{C}\left(\sum_{\kappa \in \tau_{h}} k \gamma_{n, \kappa}^{2}\right)^{1 / 2}\left\{\left\|\mathbf{f}\left(t_{n+1}\right)-\rho_{h} \mathbf{f}\left(t_{n+1}\right)\right\|_{L^{2}\left(t_{n}, t_{n+1} ; L^{2}(\Omega)^{3}\right)}+\left\|\mathbf{f}-\mathbf{f}\left(t_{n+1}\right)\right\|_{L^{2}\left(t_{n}, t_{n+1} ; L^{2}(\Omega)^{3}\right)}\right. \\
& +\left\|\mathbf{f}\left(t_{n+1}\right)-\mathbf{f}\left(t_{n}\right)\right\|_{L^{2}\left(t_{n}, t_{n+1} ; L^{2}(\Omega)^{3}\right)}+\left\|\mathbf{u}^{\prime}-\mathbf{u}_{h}^{\prime}\right\|_{L^{2}\left(t_{n}, t_{n+1} ; L^{2}(\Omega)^{3}\right)}+\left\|\nabla\left(p-p_{h}\right)\right\|_{L^{2}\left(t_{n}, t_{n+1} ; L^{2}(\Omega)^{3}\right)} \\
& \left.+\left\|\mathbf{c u r l}\left(\mathbf{u}-\mathbf{u}_{h}\right)\right\|_{L^{2}\left(t_{n}, t_{n+1} ; L^{2}(\Omega)^{3}\right)}\right\}
\end{aligned}
$$

which leads to the result.

Theorem 5.10. Finally, the pressure and the velocity verify the upper bound:

$$
\begin{aligned}
&\left\|\nabla\left(p-p_{h}\right)\right\|_{L^{2}\left(0, T ; L^{2}(\Omega)^{3}\right.}^{2}+\left\|\mathbf{u}-\mathbf{u}_{h}\right\|_{L^{\infty}\left(0, T ; L^{2}(\Omega)^{3}\right)}^{2}+\left\|\mathbf{c u r l}\left(\mathbf{u}-\mathbf{u}_{h}\right)\right\|_{L^{2}\left(0, T ; L^{2}(\Omega)^{3}\right)}^{2}+\left\|\mathbf{u}^{\prime}-\mathbf{u}_{h}^{\prime}\right\|_{L^{2}\left(0, T ; L^{2}(\Omega)^{3}\right)}^{2} \\
& \leq c\left\{\sum _ { n = 0 } ^ { N - 1 } \sum _ { \kappa \in \tau _ { h } } \left(\left(\eta_{n, \kappa}^{2}+\xi_{n, \kappa}^{2}+k \gamma_{n, \kappa}^{2}+k \delta_{n, \kappa}^{2}\right)+\left\|\mathbf{f}-\mathbf{f}^{k}\right\|_{L^{2}\left(t_{n}, t_{n+1} ; L^{2}(\kappa)^{3}\right)}^{2}\right.\right. \\
&+h_{\kappa}^{2}\left\|\operatorname{div} \mathbf{f}^{k}\right\|_{L^{2}\left(t_{n}, t_{n+1} ; L^{2}(\kappa)^{3}\right)}^{2}+\sum_{e \in \varepsilon_{\kappa}} h_{e}\left\|\left[\left(\mathbf{f}^{k}-\rho_{h} \mathbf{f}^{k}\right) \cdot \mathbf{n}\right]\right\|_{L^{2}\left(t_{n}, t_{n+1} ; L^{2}(e)\right)}^{2} \\
&\left.\left.+\left\|\mathbf{f}-\mathbf{f}\left(t_{n+1}\right)\right\|_{L^{2}\left(t_{n}, t_{n+1} ; L^{2}(\kappa)^{3}\right)}^{2}+k h_{\kappa}^{2}\left\|\rho_{h} \mathbf{f}\left(t_{n+1}\right)-\mathbf{f}\left(t_{n+1}\right)\right\|_{0, \kappa}^{2}\right)\right\}
\end{aligned}
$$

and the lower bounds defined by (5.7), (5.13), (5.32) and (5.34).

\section{REFERENCES}

[1] H. Abboud, F. El Chami \& T. Sayah A priori and a posteriori estimates for the Stokes problem with some different boundary conditions, submitted to "Numerical Algorithms"

[2] I. BABus̃ KA \& W.C. Rheinboldt A posteriori eror estimates for the finite element method, Internat. J. Numer. Meth. Engrg. 12 (1978), 1597-1615

[3] I. Babũ̃ KA \& W.C. Rheinboldt Error estimates for adaptive finite element computations, SIAM J. Numer. Anal. 15 (1978), 736-754

[4] R.E. Bank \& B.D. Welfert A posteriori eror estimates for the Stokes problem,SIAM J. Numer. Anal. 28, No. 3 (1991), 591-623

[5] C. Bègue, C. Conca, F. Murat \& O. Pironneau Les équations de Stokes et de Navier-Stokes avec des conditions aux limites sur la pression, in Nonlinear Partial Differential Equations and their Applications. Collège de France Seminar 9 (H. Brézis E6 J. L. Lions ed). Pitman Research Notes in Math. 181. Longman, Harlow, pp. 179-264 (1988)

[6] A. Bendali, J. M. Dominguez \& S. Gallic A variational approach for the vector potential formulation of the Stokes and Navier-Stokes problems in three dimensional domains, J. Math. Anal. Appl. 107 (1985), 537-560

[7] G. C. Bernardi Méthodes d'éléments finis mixtes pour les équations de Nvier-Stokes, Thèse Univ. Paris VI (1989)

[8] G. C. Bernardi Méthodes d'éléments finis mixtes pour les équations de Nvier-Stokes, Thèse Univ. Paris VI (1989)

[9] C. Bernardi \& R. Verfurth A Posteriori error analysis of the fully discretized time-dependent Stokes equations, M2AN 38, pp. 437-455 (2004)

[10] P. ClÉment Approximation by finite element functions using local regularisation.,R.A.I.R.O. Anal. Numer. 9,pp, 77$84(1975)$

[11] M. DAuge Problème de Naumann et de Dirichlet sur un polyèdre dans $\mathbb{R}^{3}$ : régularité dans des esp[aces de Sobolev $L^{p}$, C.R.A.S. 307,I , pp,27-32 (1988)

[12] F. Dubois Discrete vector potential representation of a -free vector field in three dimensional domains: numerical analysis of a model problem, Report numéro 163. Centre Math. Appl. Ecole Polytechnique (1987)

[13] G. Duvaut \& J.L.Lions Inequalities in Mechanics and Physics, Series of Comp. Studies in Math. Springer Verlag (1976) 
[14] L. Franca \& T.J. Hugues A new finite element formulation for computational fluid dynamics: VII. The Stokes Problem with various well-posed boundary conditions : symmetric formulations that converge for all velocity/pressure spaces., Comp. Meth. in Applied Mech. and Eng. 65 pp. 85-96 (1987)

[15] V. Girault \& P.A. Raviart Finite Element Methods for Navier-Stokes Equations, Springer Series in Comp. Math., 5, Springer-Verlag (1986)

[16] V. GiRAulT Curl-conforming finite element methods for Navier-Stokes equations with non-standard boundary conditions in $\mathbb{R}^{3}$, Publication du laboratoire Jacques Louis Lions, Université Pierre et Marie Curie

[17] V. GiRAult Incompressible finite element methods for Navier-Stokes equations with nonstandard boundary conditions in $\mathbb{R}^{3}$, MAth. of Comp. 51, numéro 183, pp. 55-74 (1988)

[18] P. Grisvard Behavior of the solutions of an elliptic boundary value problem in a polygonal or polyhedral domain. Numerical Solution of Partial Differential Equation III. Synspade. (B. Hubbard ed). Acadenic Press. (1975)

[19] F. Hecht \& O. Pironneau FreeFem++, see: http://www.freefem.org

[20] J. C. NedeleC Mixed finite element in $\mathbb{R}^{3}$. Numer. Math. 35, pp. 315-341 (1980).

[21] J. C. NEDELEC Eléments finis mixtes incompressibles pour l'équation de Stokes dans $\mathbb{R}^{3}$. Numer. Math. 39, pp. 97-112 (1982).

[22] J.T. Oden, W. Wu \& M. Ainthworth An a posteriori eror estimate for finite element approximations of the Navier-Stokes equations, Comput. Methods Appl. Mech. Engrg. 111 (1994), 185-202

[23] C. PADra A posteriori error estimators for nonconforming approximation of some quasi-newtonian flows, SIAM J. Numer. Anal. 34 (1997), 1600-1615

[24] P.-A. Raviart \& J.-M. Thomas A mixed finite element method for second order elliptic problems, Mathematical Aspects of Finite Element Methods. Springer, Berlin. Lect. Notes Math., 606 pp 292-315 (1977)

[25] S. REPIN A posteriori error estimates for approximate solutions of variational problems , Proc. 2nd European Conference on Numerical Mathematics and Applications, Heidelberg, Sept. 25-Oct. 3, 1997

[26] S. REPIN A posteriori estimates for the Stokes problem, J. Math. Sci. (New York), 109 (2002), 5, pp 1950-1964

[27] S. REPIN Local a posteriori estimates for the Stokes problem, Zap. Nauchn. Sem. S.-Peterburg. Otdel. Mat. Inst. Steklov. (POMI) 318 (2004), pp 233-245

[28] S. REpin \& R. Stenberg A posteriori error estimates for the generalized Stokes problem, J. Math. Sci. (New York), 142(2007), 1, pp 1828-1843

[29] R. VerfürTh Mixed finite element approximation of the vector potential, Numer. Math. 50 (1987), 685-695

[30] R. VERFüRTH A posteriori eror estimators for the Stokes equations, Numer. Math. 55 (1989), 309-326

[31] R. VERFüRTH A posteriori eror estimators for the finite element discretizations of the heat equation, $C A L C O L O 44$ (2003), 195-212 